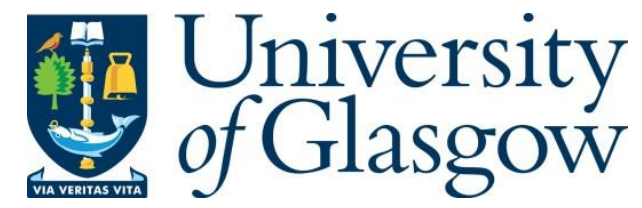

Daly, L. et al. (2017) Crystallography of refractory metal nuggets in carbonaceous chondrites: a transmission Kikuchi diffraction approach. Geochimica et Cosmochimica Acta, 216, pp. 42-60.

(doi:10.1016/j.gca.2017.03.037)

This is the author's final accepted version.

There may be differences between this version and the published version. You are advised to consult the publisher's version if you wish to cite from it.

http://eprints.gla.ac.uk/141174/

Deposited on: 31 May 2017

Enlighten - Research publications by members of the University of Glasgow http://eprints.gla.ac.uk 


\title{
Crystallography of refractory metal nuggets in carbonaceous chondrites: A transmission Kikuchi diffraction approach.
}

\author{
Luke Daly ${ }^{\mathrm{a}, *}$, Phil A. Bland ${ }^{\mathrm{a}}$, Kathryn A. Dyl ${ }^{\mathrm{a}}$, Lucy V. Forman ${ }^{\mathrm{a}}$, David W Saxey ${ }^{\mathrm{b}}$, Steven M. Reddy ${ }^{\mathrm{a}, \mathrm{b}}$, \\ Denis Fougerouse ${ }^{\mathrm{a}, \mathrm{b}}$, William D. A. Rickard ${ }^{\mathrm{b}}$, Patrick W. Trimby ${ }^{\mathrm{c}}$, Steve Moody ${ }^{\mathrm{c}}$, Limei Yang $^{\mathrm{c}}$, Hongwei \\ Liu $^{c}$, Simon P. Ringer ${ }^{d}$, Martin Saunders ${ }^{\text {, Sandra Piazolo }}{ }^{\text {f }}$ \\ ${ }^{a}$ Department of Applied Geology, Curtin University, GPO Box U1987, Perth, WA 6845, Australia. \\ ${ }^{b}$ Geoscience Atom Probe Facility, Advanced Resource Characterisation Facility, John de Laeter Centre, Curtin University, \\ GPO Box U1987, Perth, WA, Australia. \\ ${ }^{c}$ Australian Centre for Microscopy and Microanalysis, The University of Sydney, NSW 2006, Australia. \\ ${ }^{d}$ Australian Institute for Nanoscale Science and Technology, and School of Aerospace, Mechanical and Mechatronic \\ Engineering, The University of Sydney, NSW, 2006, Australia. \\ ${ }^{e}$ Centre for Microscopy, Characterisation and Analysis, The University of Western Australia, WA 6009, Australia. \\ ${ }^{f}$ Department of Earth and Planetary Sciences, Macquarie University NSW, 2109, Australia.
}

\section{Abstract}

Transmission Kikuchi diffraction (TKD) is a relatively new technique that is currently being developed for geological sample analysis. This technique utilises the transmission capabilities of a scanning electron microscope (SEM) to rapidly and accurately map the crystallographic and geochemical features of an electron transparent sample. TKD uses a similar methodology to traditional electron backscatter diffraction (EBSD), but is capable of achieving a much higher spatial resolution (5-10 nm) (Trimby, 2012; Trimby et al., 2014). Here we apply TKD to refractory metal nuggets (RMNs) which are micrometre to sub-micrometre metal alloys composed of highly siderophile elements (HSEs) found in primitive carbonaceous chondrite meteorites. TKD allows us to analyse RMNs in situ, enabling the characterisation of nanometre-scale variations in chemistry and crystallography, whilst preserving their spatial and crystallographic context. This provides a complete representation of each RMN, permitting detailed interpretation of their formation history.

We present TKD analysis of five transmission electron microscopy (TEM) lamellae containing RMNs coupled with EBSD and TEM analyses. These analyses revealed textures and relationships not previously observed in RMNs. These textures indicate some RMNs experienced annealing, forming twins. Some RMNs also acted as nucleation centres, and formed immiscible metal-silicate fluids. In fact, each RMN analysed in this study had different crystallographic textures. These RMNs also had heterogeneous compositions, even between RMNs contained within the same inclusion, host phase and even separated by only a few nanometres. Some RMNs are also affected by secondary processes at low temperature causing exsolution of molybdenite. However, most RMNs had crystallographic textures indicating that the RMN formed prior to their host inclusion. TKD analyses reveal most RMNs have been affected by processing in the protoplanetary disk. Despite this alteration, RMNs still preserve primary crystallographic textures and heterogeneous chemical signatures. This heterogeneity in crystallographic relationships, which mostly suggest that RMNs pre-date

\footnotetext{
${ }^{*}$ Corresponding author: Luke Daly, Email: luke.daly@postgrad.curtin.edu.au, telephone: +61497840194
} 
their host, is consistent with the idea that there is not a dominant RMN forming process. Each RMN has experienced a complex history, supporting the suggestion of Daly et al. (this issue), that RMNs may preserve a diverse pre-solar chemical signature inherited from the Giant Molecular Cloud.

Keywords: Refractory Metal Nuggets, Solar Nebula, Solar System, Origin, Meteorites, carbonaceous chondrites, crystallography, crystallisation, Transmission Kikuchi

diffraction

\section{Introduction}

There are two main hosts for highly siderophile elements (HSEs) in primitive carbonaceous chondrite meteorites: refractory metal nuggets (RMNs) and opaque assemblages (OAs). OAs are larger ( $>10 \mu \mathrm{m})$ multi-phase alloys (El Goresy et al., 1977), whereas RMNs are single phase alloys. OAs originally were 5 termed fremdlinge due to their proposed pre-solar origin (El Goresy et al., 1977). However, isotopic analysis of fremdlinge by Hutcheon et al. (1987) indicate that they have solar isotopic ratios and so were named OAs by Palme et al. (1994) to reflect their solar origin.

To avoid confusion between the two objects we define RMNs in this study as any micrometre-submicrometre homogeneous (Harries et al. 2012) metal alloy within carbonaceous chondrite meteorites which contain $>1$ atomic percent at. \% ) HSEs (Re, Os, Ir, Pt, Ru, Rh, W or Mo), in line with the definition in Daly et al. (this issue).

Since the co-discovery of RMNs by Palme \& Wlotzka (1976) and Wark \& Lovering (1976), it had been assumed that they are only found in Ca-Al rich inclusions (CAIs) (Blander et al., 1980, Berg et al., 2009, Harries et al., 2012, Hewins et al., 2014). However, observations of RMNs in chondrules (Wang et al., 2007), matrix (Schwander et al. 2015a), an unusual xenolith from Allende, likely to be a dark inclusion (Palme et al. 1989), as well as recent X-ray fluorescence microscopy (XRF) synchrotron data, have revealed that this is not the case. Daly et al. (this issue) showed that RMNs are found within all components of carbonaceous chondrites. The refractory nature of RMNs which contain only elements with $50 \%$ condensation temperatures above Fe and Ni (Lodders, 2003) lead to the conclusion that RMNs are primary condensates „Berg et al., 2009, Fegley \& Palme, 1985, Grossman, 1973, Harries et al., 2012, Lodders, 2003; Palme \& Wlotzka, 1976). This is consistent with the assumption that RMNs were hosted solely within CAIs (Berg et al. 2009: Harries et al., 2012, Hewins et al., 2014; Blander et al., 1980) which also form at high temperatures (MacPherson et al. 2005; Grossman et al., 2000) and are thought to form initially as condensates (Grossman, 1973). Thus, formation mechanisms for RMNs were only considered within the context of CAIs.

Recent work by Schwander et al. (2015a) and Rudraswami et al. (2014) respectively has shown that this is not necessarily the case: an alternative mechanism may be crystallisation of RMNs from a silicate melt, CAI melt, or during the formation of cosmic spherules. Furthermore, RMNs have, in some cases, been subjected to secondary processes as implied by sulphidation and oxidation textures (Bischoff \& Palme, 1987. Blum et al. 1988, Palme \& Wlotzka, 1976). However, other studies have suggested that RMNs may have 
a pre-solar origin (El Goresy et al., 1977, 1978, Wark \& Lovering, 1976). This was confirmed by recent observations of 20 - $50 \mathrm{~nm}$ RMNs hosted within pre-solar graphite (Croat et al., 2013).

A combination of 113 in situ energy-dispersive X-ray spectroscopy (EDS) measurements of RMNs and the database of RMNs reported in the literature over the last 40 years, revealed a large range of RMN compositions which cannot be reconciled by a single Solar System process (Daly et al. this issue). Our interpretation is that an inherently diverse population of RMNs was incorporated into the protoplanetary disk. These RMNs were altered by subsequent disk processing, but the primordial diversity was never overprinted (Daly et al., this issue).

The crystallography of RMNs can offer important insights into their origin and formation. For example, the three phase condensation model of Sylvester et al. (1990) predicted that RMNs should be found in three main crystal systems: body centred cubic (bcc) composed predominantly of W, Mo, and Cr; hexagonal close packed (hcp) composed predominantly of Re, Os, and Ru; and a face centred cubic (fcc) structure composed of Ir, Pt, Rh, Co, Ni, Fe, and Au. This assertion was proposed to reconcile RMN compositions that do not fit a single-phase condensation model. However, the observed crystallography of RMNs using transmission electron microscopy (TEM) spot diffraction patterns indicate that they are largely monocrystalline, homogeneous, subhedral-euhedral hcp crystals (Eisenhour \& Buseck, 1992, Harries et al., 2012, Croat et al., 2013). This observation does not support the three phase condensation model, and reinforces the idea of a single phase equilibrium condensation origin for RMNs, in agreement with calculations by Campbell et al. (2001) and Wark \& Lovering (1976). However, the chemical heterogeneity of RMNs is still not fully understood. []

Previous work has either been limited to analysis of a few grains in situ (Blum et al., 1988, El Goresy et al., 1978, Palme \& Wlotzka, 1976, Wark, 1986, Wark \& Lovering, 1976), or analysis of many grains using acid residues (Berg et al., 2009, Harries et al., 2012, Schwander et al., 2014) where the original context of the RMN was lost. Two studies have analysed large quantities of RMNs in situ: Daly et al. (this issue) and Schwander et al. (2015b). Schwander et al. (2015b) demonstrated that the condensation temperature of the host mineral phase did not correlate with appropriate RMN compositions assuming RMNs formed as condensates, implying that condensation was an unlikely formation mechanism for RMNs in that study.

Analyses of inclusion-host systems in terrestrial rocks using large electron backscatter diffraction (EBSD) datasets, and the documentation of crystallographic orientation relationships (CORs) between mineral phases by Griffiths et al. (2016) revealed variable CORs between mineral inclusions and their host phase relate to changing metamorphic conditions. The context of the RMN is integral to the accurate interpretation and discernment of mineral formation mechanisms, as the formation history of the host itself will influence the interpretation. For example, RMNs hosted within chondrules which are not thought to have a condensation history, may indicate that these RMNs formed via a different mechanism. Also, any secondary alteration events experienced by the host phase would necessarily affect the RMN and may influence its crystallography and composition.

To determine which processes have affected RMNs since their formation, high resolution in situ analyses 
are required. Ma et al. (2014) demonstrated that combining chemical and crystallographic data through EBSD can be used to determine the origins of refractory grains such as RMNs, interpreting two new mineral phases, allendeite and hexamolybdenum, as primary nebular condensates. In this work we will apply transmission Kikuchi diffraction (TKD); a relatively new technique for which the applications to geological (Jacob et al. 2016, Piazolo et al. 2016) and meteoritical samples (Forman et al., 2016) are beginning to be explored. TKD is also referred to as transmission EBSD (t-EBSD) (Keller \& Geiss, 2012). The technique allows the elemental and crystallographic mapping of a TEM lamella in a scanning electron microscope (SEM). It uses the same software and techniques as EBSD, but with an order of magnitude improvement in the resolution attainable (Trimby, 2012, Trimby et al. 2014, Sneddon et al., 2016). This approach allows us to observe RMNs in context, preserving any CORs with associated host phases, and rapidly map the crystallography of these grains at $\sim 5 \mathrm{~nm}$ resolution. It therefore provides a completely new window on the crystallography, orientation, and deformation history of these materials that has not been accessible before. TKD analysis can facilitate better interpretation of the sequence of events that may have affected each RMN since formation, and how these processes may have affected the RMNs crystallography and chemistry.

The only other study utilising TKD on meteoritic materials by Forman et al. (2016) demonstrated that TKD can be used to highlight the differences in how matrix and chondrules are affected by temperature and stress during impact induced compaction in the matrix of Allende near a chondrule. Here we will further demonstrate the potential of this technique in the analysis of RMNs. We will show and explore the possible observable effects on the crystallography and chemistry, produced by primary nebula processes, i.e. condensation and crystallisation; secondary nebular processes, i.e. transient heating events; and secondary asteroidal processes, i.e. sulphidation. TKD observations are supplemented with the utilisation of established techniques, such as EBSD and TEM.

\section{Methods}

To identify RMNs in situ, a combination of Synchrotron XRF analyses corroborated with SEM observations using map overlay and feature mapping software packages was used. A full description of the procedure can be found in Daly et al. (this issue). Several RMN bearing regions were identified for analysis in this study: Region A1, a sulphide nodule hosted within a porphyritic olivine (PO) chondrule in Allende; A2 and A3 were found within different forsterite enclaves within the same spinel- diopside-rich Type B CAI in Allende; ALH1, was extracted from a type B-like CAI in ALH 77307; and V1 and V2 from the reheated portion of a 'fluffy' type A CAI in Vigarano. Backscattered electron maps relating to the wider petrographic context of each RMN in this study is provided in Supplementary material A. Regions A1 and A3 were analysed using EBSD techniques. Ideally all regions would have been analysed by EBSD, however, these were the only regions where a sufficient polish was achieved to undertake EBSD. Regions A1, A2, ALH1, V1, and V2 were extracted and analysed using TKD, and region ALH1 was also analysed using TEM. 
The samples were prepared for EBSD by hand polishing for 30 minutes using $0.01 \mu \mathrm{m}$ colloidal silica in $\mathrm{NaOH}$ solution. This reduces the risk of polishing away the micrometre sized RMNs identified on the surface whilst achieving the polish required for EBSD. The samples were then coated with $10 \mathrm{~nm}$ of carbon. EDS and EBSD data were collected using the Zeiss ULTRA Plus Field emission gun (FEG) SEM at the Australian Centre for Microscopy and Microanalysis (ACMM), The University of Sydney, with an X-Max $20 \mathrm{~mm}^{2}$ SDD EDS detector at an accelerating voltage of $20 \mathrm{kV}$, and probe current of $4-20 \mathrm{nA}$.

\subsection{Focussed Ion Beam}

The samples A1, A2, ALH1, V1, and V2 were extracted and prepared for TEM and TKD using a focussed ion beam (FIB) on the Zeiss Auriga FIB-SEM at the ACMM, The University of Sydney. The regions of interest were initially covered with a protective strip of Pt using secondary electrons, followed by a thicker layer deposited using the Ga beam. This procedure is to protect the sample during milling, and thinning, while also adding structural support to the lamellae. The section was extracted using a Kleindiek micromanipulator, and welded to a Cu TEM grid with further Pt deposition. The sample was then thinned to approximately $100 \mathrm{~nm}$ thickness to allow the transmission of electrons.

The samples A1, A2, ALH1, V1, and V2 were analysed using TKD on the Zeiss ULTRA Plus FEG SEM at the ACMM, The University of Sydney. TKD mapping was performed using a Nordlys-NANO EBSD detector, and EDS maps were collected with an X-Max $20 \mathrm{~mm}^{2}$ SDD EDS detector using an acceleration voltage of $30 \mathrm{kV}$ as described in Trimby (2012) and Trimby et al. (2014).

\subsection{Data reduction}

The EDS, EBSD and TKD maps were collected and interpreted using the Oxford instruments AZtec and Channel 5 software packages. Grain boundaries were defined by $>10$ degree misorientation. Element abundances were derived from the EDS maps using standardless quantitative methods embedded in the Aztec software. EDS measurements of RMNs were taken with a process time of 5 . This allows for better energy resolution and detection limits. The L-alpha X-Ray lines were used to calculate elemental abundances for $\mathrm{Ru}, \mathrm{Mo}, \mathrm{W}, \mathrm{Re}, \mathrm{Os}$, Ir and Pt, as these are better separated than the M lines. K X-ray lines were used to calculate Fe and Ni abundances. Detection limits for elements within RMNs were approximately $<1$ at. $\%$. The relative uncertainty in these measurements is approximately $\pm 10 \%$. We also assessed the accuracy of the EDS measurement by comparing EDS and atom probe microscopy measurements from a $1 \mathrm{~mm}$ RuIrOs nugget from a placer deposit in Tasmania, Australia. All elements were consistent between EDS and atom probe within the $10 \%$ relative uncertainty except for Ir which was within $13 \%$ (see Supplementary Material B for a full description). This provides sufficient precision to detect major and minor elements and draw comparisons between measured RMN compositions in different meteoritic components. All other elements 
present in the spectra such as $\mathrm{Mg}, \mathrm{Al}, \mathrm{Ca}, \mathrm{Cu}$ etc; were ignored following the methodology of Schwander

al. (2015b) and Daly et al. (this issue), as they are likely to be generated by fluorescence in the SEM chamber from elements in the surrounding phases, or the sample mount. We do not expect these elements to be present in RMNs, but for completeness the full compositions calculated for each analysis including the contaminating elements is included in Supplementary Material C.

Not all expected HSEs were detected in every EDS analysis. For example, Rh was only detected in one RMN which may be due to its low abundance (Lodders, 2003). Other HSEs may be present in the RMNs in concentrations below the detection limits of the EDS.

RMNs were mostly characterised by a fcc awaruite or a hcp rutheniridosmine crystal structure from the EBSD database as the phase that best described most RMNs observed.

\subsection{TEM}

The FIB lamella taken from ALH 77307 (region ALH1) was also analysed using the JEOL2200 TEM, ACMM, the University of Sydney and the FEI Titan G280 - 200 TEM/STEM, Centre for Microscopy, Characterisation and Analysis (CMCA), University of Western Australia at an accelerating voltage of 200 $\mathrm{kV}$. The sample was mounted on a double-tilt sample holder allowing the sample to be tilted in two directions. This allows greater flexibility to find and identify crystallographic axes. High resolution EDS maps, spot diffraction patterns and high resolution TEM (HRTEM) images were collected.

\section{Results}

\subsection{Allende - region A1}

This region revealed RMNs hosted within a pentlandite nodule. The pentlandite nodule is hosted in the rim of a sulphide-rich PO chondrule. Three RMNs were observed on the surface in the EBSD dataset (Figure 1), while a further five RMNs were revealed during the TKD analyses that were previously buried beneath the surface of the sample (Figure 2). The RMNs varied in size from $0.1 \mu \mathrm{m}$ to $1 \mu \mathrm{m}$, and form anhedral-subhedral fcc single crystals. The RMNs had identical CORs relative to each other (Figures 1 and 2). The RMNs have no COR with the pentlandite host phase, however, they do share a [100] axis with the nearby forsterite crystal (Figure 1 and 2). Three RMNs from the EBSD dataset (Table 1 ) and four from the TKD dataset (Table 2) were large enough to generate good EDS spectra. The composition of the RMNs indicated that in most cases $\mathrm{Fe}$ and $\mathrm{Ni}$ are relatively homogeneous between RMNs, with the exception of entry \# 3 in Table 2, Fe and Ni were overestimated in the EBSD dataset as the interaction volume of the EDS measurement was larger than the RMN. This means that there was a contribution to the EDS spectra from the surrounding pentlandite. The abundances of Ir and Pt vary between RMNs (Tables 1 and 2). The Pt should be interpreted with caution as there was likely to be some contribution of Pt from the fluorescence of the Pt protective layer deposited during FIB sample preparation in the EDS spectra in Table 2. However, 
the EDS spectra from the EBSD dataset (Table 1) confirms that Pt is present and variable. Mo and W are completely absent in all RMNs in this region and Os is only observed in RMN 2 in Table 2).

Table 1: RMN compositions from EDS analysis from Figure 1 The RMN number relates to the numbered regions of Figure 1 b. b.d. = below detection limit: 0.2 at. \% Ir.

\begin{tabular}{ccccc}
\multirow{2}{*}{ RMN } & \multicolumn{4}{c}{ at. \% } \\
& $\mathrm{Fe}$ & $\mathrm{Ni}$ & $\mathrm{Ir}$ & $\mathrm{Pt}$ \\
\hline 1 & 62.2 & 30.8 & 6.1 & 0.9 \\
2 & 61.3 & 32.9 & b.d. & 5.8 \\
3 & 62.4 & 27.8 & 2.0 & 7.8 \\
\hline
\end{tabular}

Table 2: RMN compositions from EDS analysis from Figure 2 The RMN number relates to the numbered regions of Figure 2. b.d. = below detection limit: 0.1 at. $\%$ Os and 0.1 at. $\% \mathrm{Pt}$.

\begin{tabular}{cccccc}
\multirow{2}{*}{ RMN } & \multicolumn{5}{c}{ at. $\%$} \\
& $\mathrm{Fe}$ & $\mathrm{Ni}$ & $\mathrm{Os}$ & $\mathrm{Ir}$ & $\mathrm{Pt}$ \\
\hline 1 & 45.2 & 33.5 & b.d. & 1.8 & 19.6 \\
2 & 45.4 & 32.3 & 0.7 & 4.8 & 16.8 \\
3 & 29.0 & 63.6 & b.d. & 7.4 & b.d. \\
4 & 51.9 & 31.8 & b.d. & 6.7 & 9.7 \\
\hline
\end{tabular}

\subsection{Allende - region A2}

This RMN was extracted from a forsterite enclave within a spinel and diopside-rich Type-B CAI in the Allende CV3 meteorite. TKD revealed a cluster of RMNs with two distinct morphologies. The first was a large polycrystalline RMN associated with molybdenite. The second was several smaller spherical isolated RMNs $(20-100 \mathrm{~nm})$ hosted in forsterite. The whole inclusion was surrounded by spinel (Figure 3). There is no COR between the spinel and the inclusion. All RMNs here had a hep structure and uniform chemistry in relation to $\mathrm{Ru}$, Ir and Pt. They were also completely devoid of Mo which was situated in the molybdenite (Table 3). The molybdenite and the larger polycrystalline RMN are crystallographically aligned with each other. They also exhibit a higher misorientation density, whereas the forsterite and smaller RMNs are uniform single crystals. The surrounding spinel is equigranular $(2-5 \mathrm{\mu m})$ with $120^{\circ}$ grain boundary intersections.

\subsection{Allende - region A3}

This RMN was hosted within another forsterite enclave within the same spinel-, diopside-rich Type-B CAI as A2. EBSD analyses revealed that the RMN has a COR with forsterite, sharing the [110] axis (Figure 4). 


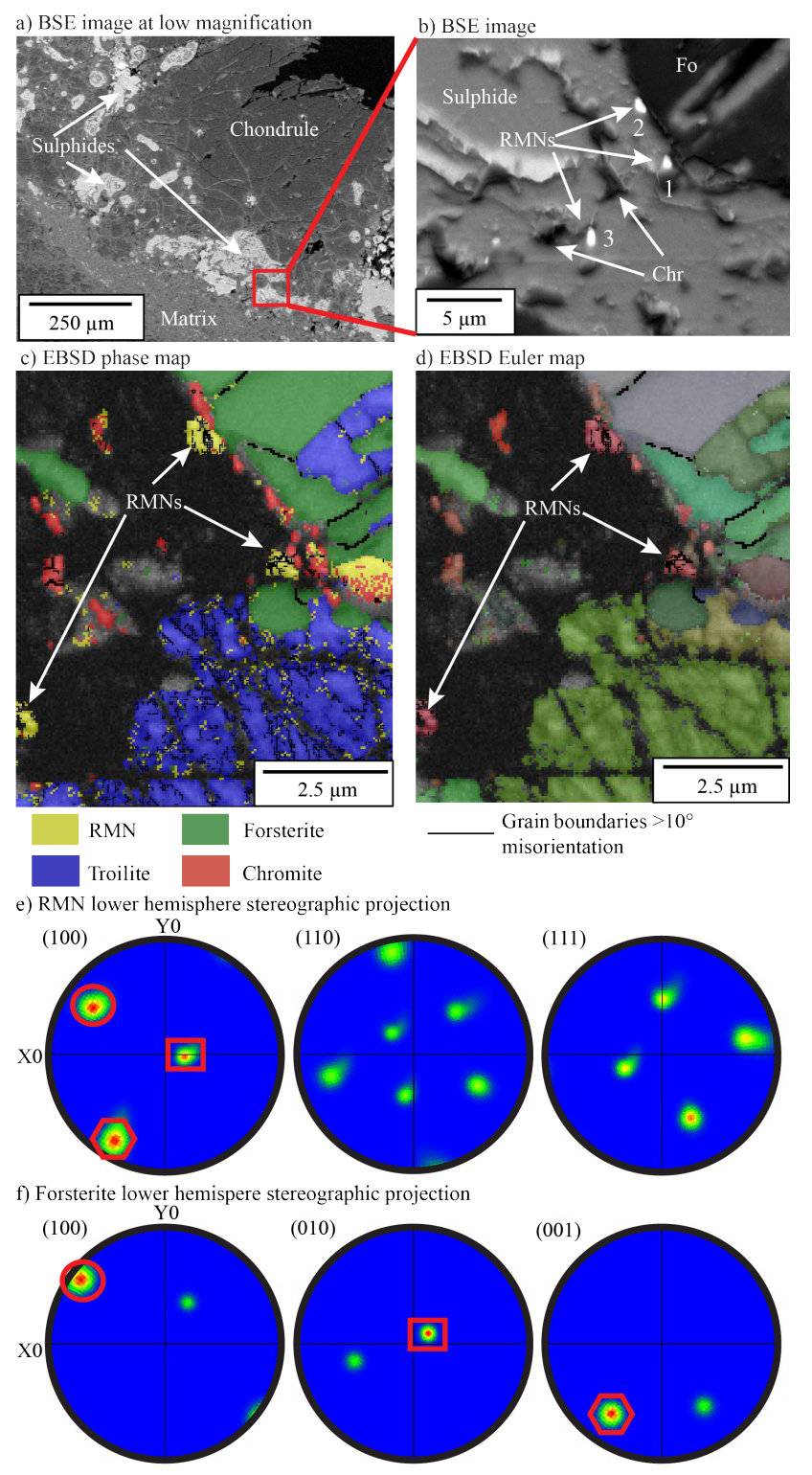

Figure 1: Characteristics of Allende - region A1: EBSD analyses of 3 RMNs are contained within a chondrule-hosted sulphide nodule. a) A low magnification back scatter electron (BSE) image of region A1, indicating the wider context of the chondrule host with associated sulphide nodules, b) A BSE image of the RMNs hosted within a sulphide, with a nearby forsterite grain (Fo) as well as associated Cr-spinel (Chr) inclusions. The numbers indicate where the EDS measurements were taken from in Table 1 c) An EBSD phase map overlain on a band contrast image indicating RMNs (yellow), troilite (blue) Cr-Spinel (red) and forsterite (green), the pentlandite sulphide did not index well and is located within the black area, d) An EBSD Euler image overlain on a band contrast image indicating crystallographic orientations of each phase, e) A lower hemisphere stereographic projection of RMN crystallographic axes (100), (110), and (111). The crystallographic orientations of the RMNs revealed all RMNs have the same crystallographic alignment, f) A lower hemisphere stereographic projection of the orientation of the forsterite crystallographic axes (100), (010), and (001). The red square, circle and hexagon on stereographic projections e) and f) indicate that the RMNs and the forsterite share a [100] axis. 

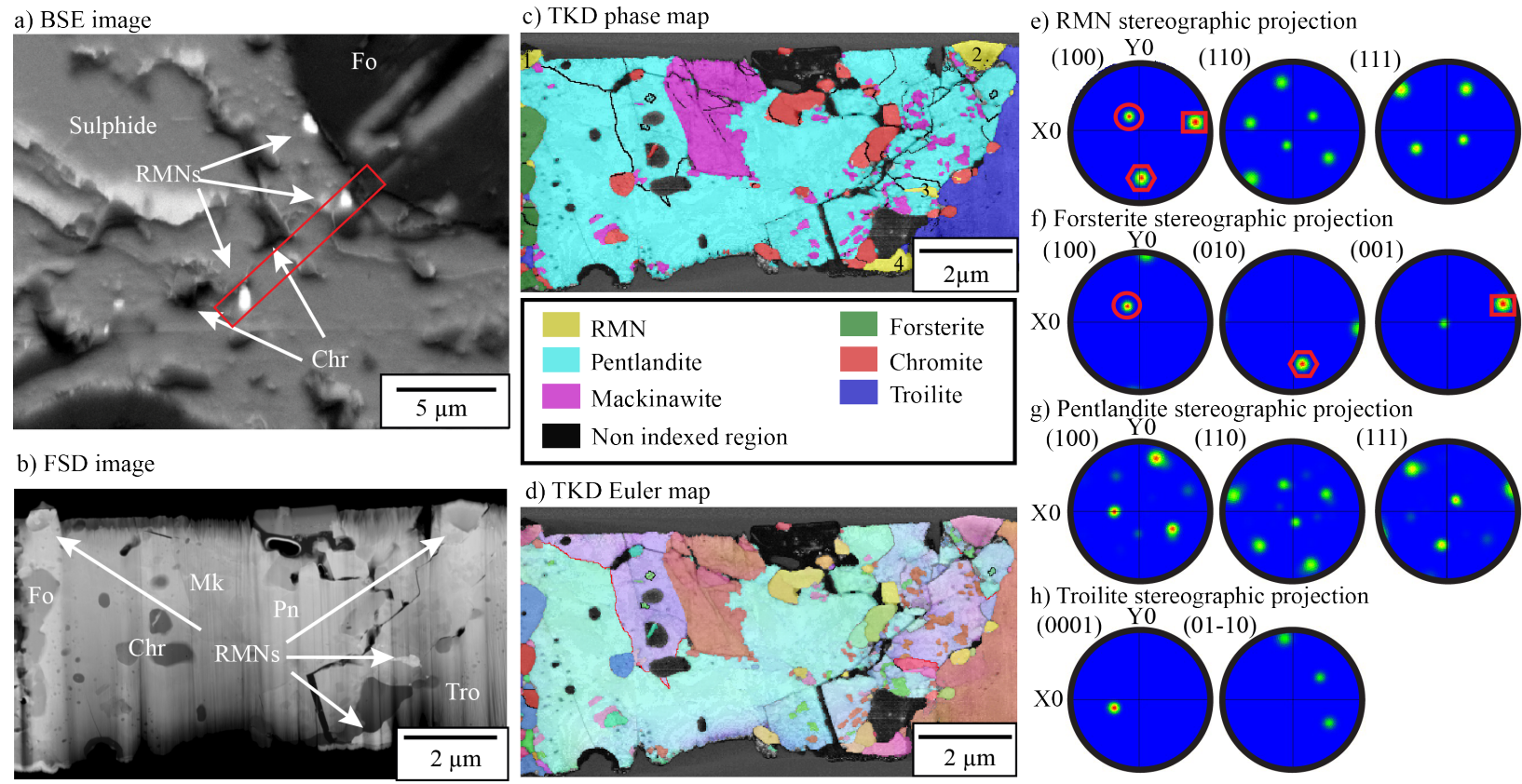

f) Forsterite stereographic projection

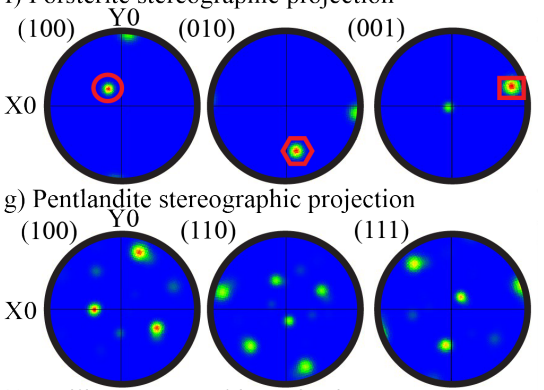

h) Troilite stereographic projection

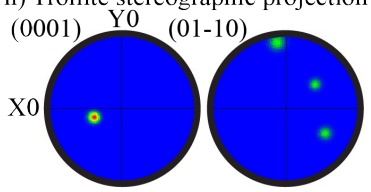

Figure 2: Characteristics of Allende - region A1: TKD analyses from a FIB-produced TEM lamellae containing an RMN bearing sulphide nodule hosted in a chondrule. a) A BSE image of the RMNs in context prior to extraction. The TEM lamellae was extracted as indicated by the red rectangle. Associated minerals are forsterite (Fo), Cr-spinel (Chr) and sulphide, b) A TKD fore-scatter detector (FSD) image of the TEM lamellae RMNs and associated minerals are labelled forsterite (Fo), mackinawite (Mk), Cr-spinel (Chr), pentlandite (Pn) and troilite (Tro), c) TKD phase map, revealing five more RMNs (yellow) which were buried beneath the surface of the sample, the numbers relate to EDS measurements detailed in Table 2 d) TKD Euler map showing crystallographic orientations of each phase in the section, e) Lower hemisphere stereographic projection of crystallographic orientations of the RMNs revealed a consistent crystallographic orientation for all RMNs, f) Lower hemisphere stereographic projection of crystallographic orientations of the forsterite confirming a shared [100] axis between the RMNs and forsterite observed in Figure 1, denoted by the red circle square and hexagon, g) Lower hemisphere stereographic projection of crystallographic orientations of pentlandite, indicating no COR between the RMN and the pentlandite h) Lower hemisphere stereographic projection of crystallographic orientations of the troilite, indicating no COR between the RMN and the troilite. 

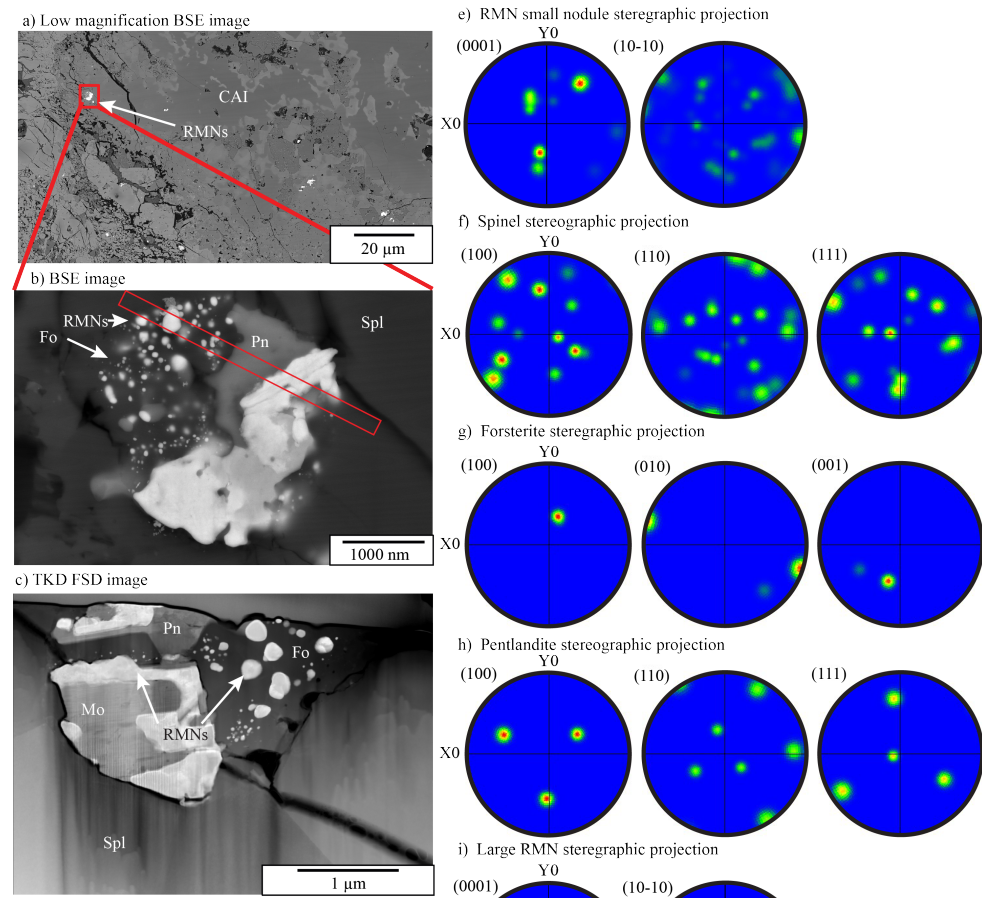

g) Forsterite steregraphic projection
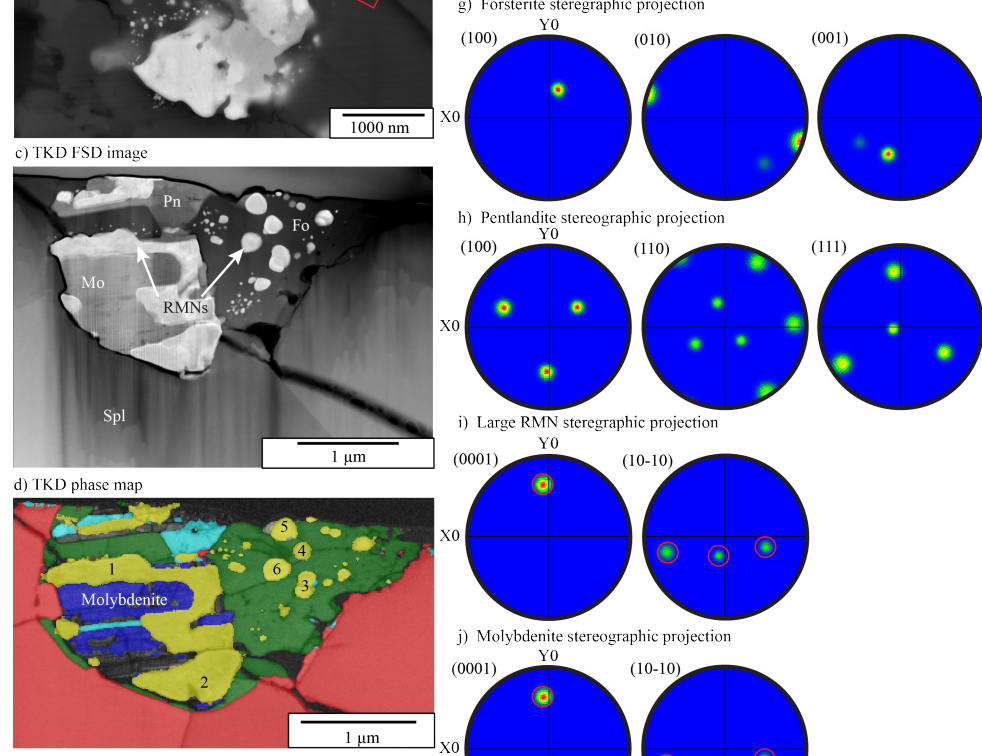

RMNs Spinel $\square$ Molybdenite

Pentlandite

Forsterite

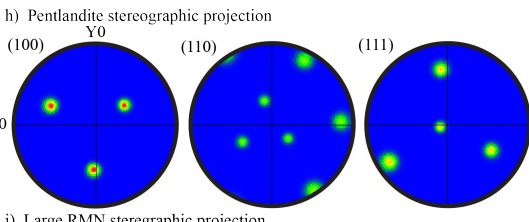

i) Large RMN steregraphic projection

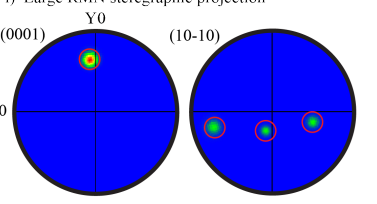

j) Molybdenite stereographic projection

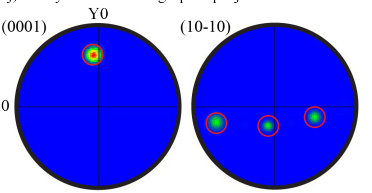

Figure 3: Characteristics of Allende region A2: TKD analyses from a FIB-produced TEM lamellae of an RMN-bearing forsterite inclusion hosted in a CAI. a) A low magnification BSE image of region A2 indicating the wider context of the CAI host, b) A BSE image of the RMNs (bright spots) in context prior to extraction. The TEM lamellae was extracted as indicated by the red rectangle. RMNs and associated minerals were labelled forsterite (Fo), pentlandite (Pn), and spinel (Spl), c) A FSD image of the TEM lamellae indicating the RMN bearing region at the top and revealing the surrounding spinel has $120^{\circ}$ grain boundary intersections and $2-5 \mu \mathrm{m}$ grain size. RMNs and associated minerals are labelled forsterite (Fo), pentlandite (Pn), molybdenite (Mo), and spinel (Spl), d) TKD phase map, revealing a large RMN associated with molybdenite and smaller spherical RMNs suspended in forsterite. The numbers relate to EDS measurements in Table 2 The whole inclusion is encapsulated by spinel, e) Lower hemisphere stereographic projection of crystallographic orientations of the small RMN spheres revealing random crystallographic orientations for each RMN relative to each other, f) Lower hemisphere stereographic projection of crystallographic orientations of the spinel, g) Lower hemisphere stereographic projection of crystallographic orientations of the forsterite, h) Lower hemisphere stereographic projection of crystallographic orientations of the pentlandite, i) Lower hemisphere stereographic projection of crystallographic orientations of the large RMN, j) Lower hemisphere stereographic projection of crystallographic orientations of molybdenite. The molybdenite and the large RMN are crystallographically aligned parallel to one another as the indicated by the red circles in i) and $\mathrm{j}$ ). 
a) FSD image at low magnification

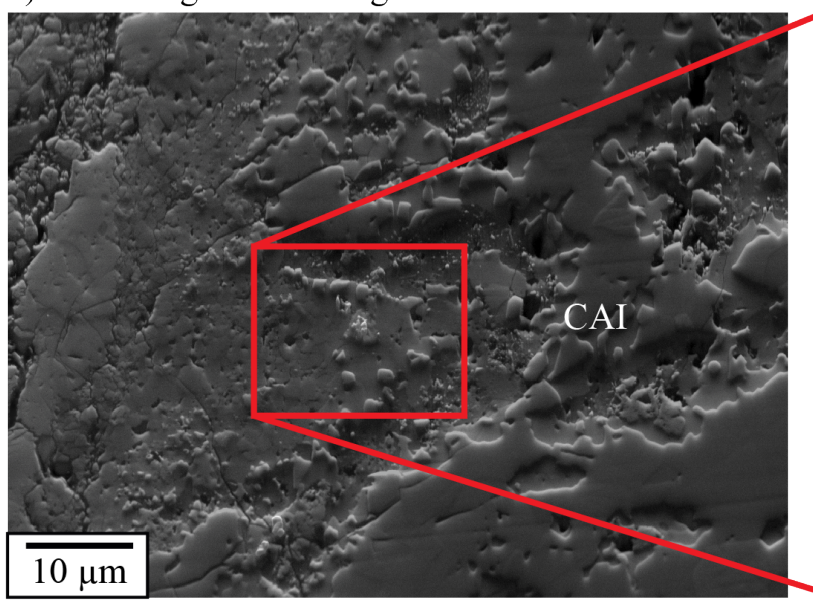

c) EBSD phase map

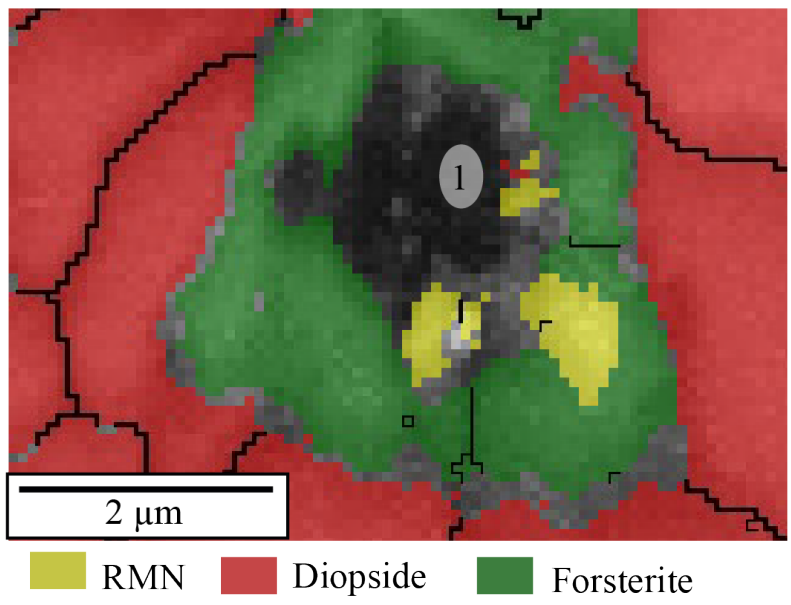

e) RMN stereographic projection

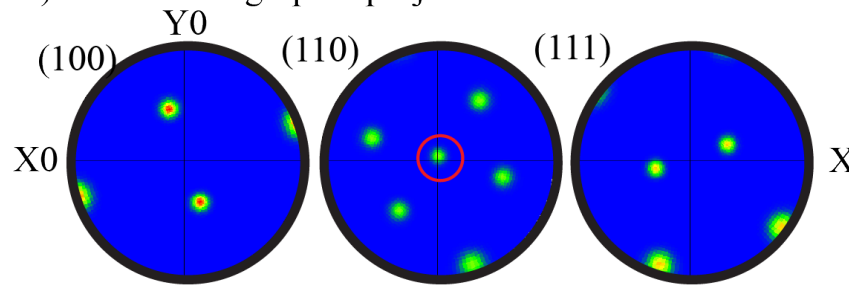

b) FSD image

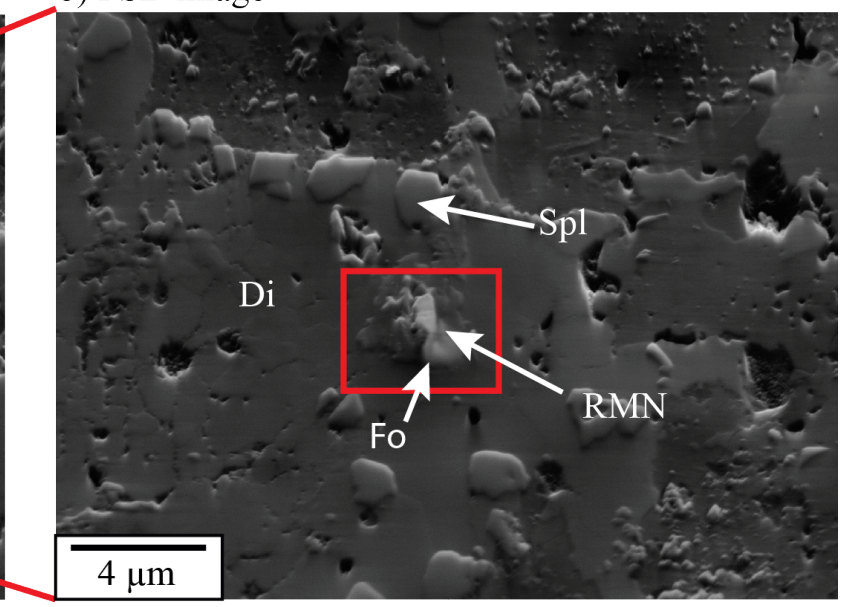

d) EBSD euler image

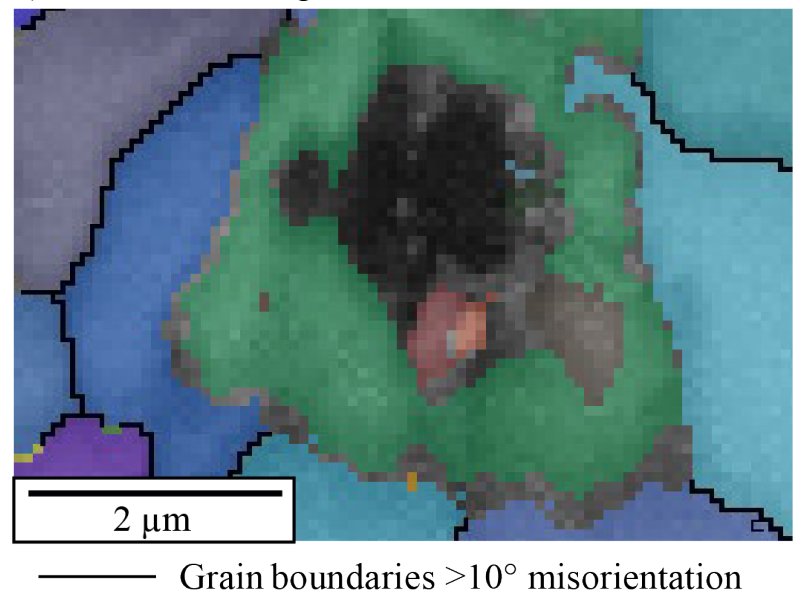

f) Forsterite stereographic projection

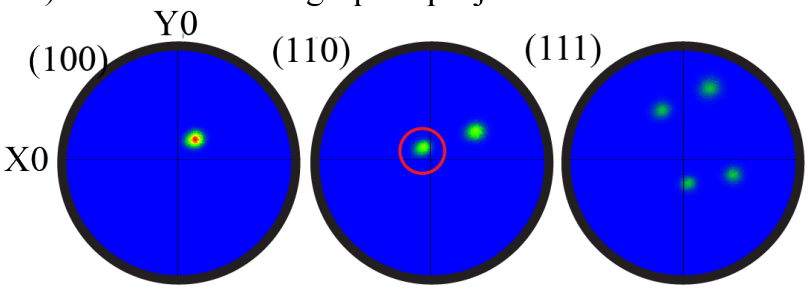

Figure 4: Characteristics of Allende - region A3: EBSD analyses of a forsterite hosted RMN from a CAI. a) A low magnification FSD image of region A3 (red box) indicating the context of the RMN within the CAI, b) FSD image of Region A3 indicating where the EBSD dataset was acquired (red box), associated minerals are labelled forsterite (Fo), spinel (Spl) and diopside (Di), c) EBSD phase map indicating an RMN hosted within a forsterite grain, the number indicates the EDS measurement taken from that point detailed in Table 4 d) Euler map indicating crystallographic orientations, e) Lower hemisphere stereographic projection of crystallographic orientations of the RMN, f) Lower hemisphere stereographic projection of crystallographic orientations of the forsterite. Comparing the projections reveals the RMN have a COR with the forsterite sharing their (110) axis (red circle). 
Table 3: RMN compositions from EDS analyses from the TEM lamellae detailed in Figure 3 The RMN number relates to the numbered regions of Figure 3r. b.d. = below detection limit: 1 at. $\% \mathrm{Ni}, 1$ at. $\% \mathrm{Mo}, 0.4$ at. \% Ru, 0.1 at. $\%$ Os, 0.2 at. $\%$ Ir and 0.6 at. $\% \mathrm{~S}$.

\begin{tabular}{cccccccc}
\hline \multirow{2}{*}{ Spectrum Label } & \multicolumn{7}{c}{ at. $\%$} \\
& Fe & Ni & Mo & Ru & Os & Ir & S \\
\hline Molybdenite & 0.9 & b.d. & 59.9 & b.d. & b.d. & b.d. & 39.2 \\
1 & 1.5 & b.d. & 2.6 & 34.0 & 32.8 & 26.1 & b.d. \\
2 & 4.6 & b.d. & 1.8 & 33.9 & 34.0 & 25.0 & b.d. \\
3 & 5.3 & b.d. & b.d. & 31.4 & 37.6 & 25.2 & b.d. \\
4 & 5.7 & b.d. & b.d. & 29.5 & 36.2 & 29.0 & b.d. \\
5 & 4.8 & b.d. & b.d. & 30.6 & 36.6 & 28.0 & b.d. \\
6 & 6.3 & b.d. & b.d. & 28.5 & 39.1 & 26.0 & b.d. \\
\hline
\end{tabular}

Table 4: RMN composition from EDS analyses from Figure 4 The RMN number relates to the numbered regions of Figure 4 F.

\begin{tabular}{ccccccc}
\multirow{2}{*}{ RMN } & \multicolumn{7}{c}{ at. \% } \\
& Fe & Ni & Ru & Mo & Os & Ir \\
\hline 1 & 21.5 & 5.3 & 50.4 & 3.8 & 4.5 & 14.6 \\
\hline
\end{tabular}

\subsection{ALH 77307 - region ALH1}

ALH1 is an RMN extracted from a perovskite bearing melilite rich type B-like CAI rimmed with diopside in ALH 77307 CO3.0. TKD and TEM analysis revealed a single RMN hosted within melilite. The RMN is polyphase with a fcc region and a trigonal region. The trigonal crystal system is an unknown configuration in RMNs. We will refer to this trigonal region henceforth as the unknown phase, to distinguish it from the regular fcc RMN. As the unknown phase had not previously been documented, no mineral EBSD data were available to index it. Therefore, a proxy of $\mathrm{Fe}_{7} \mathrm{~W}_{6}$ with space group 166 and $a=b=0.4731 \mathrm{~nm}$ and $c=2.57$ $\mathrm{nm}$ was used. The trigonal crystal structure of the unknown phase had a very long $\mathrm{c}$ axis relative to the a and $\mathrm{b}$ axes. Using this proxy and TEM spot diffraction, this phase was revealed to be closely related (mean angular deviation of 0.79 ) to the trigonal $\mathrm{Fe}_{7} \mathrm{~W}_{6}$ proxy with $a=b=0.46 \mathrm{~nm} c=2.55 \mathrm{~nm}$ with space group R$3 \mathrm{~m}(166)$, and a simplified chemical formula $(\mathrm{Fe}, \mathrm{Ni})_{6}(\mathrm{Mo}, \mathrm{Ru}, \mathrm{W}, \mathrm{Os}, \mathrm{Ir})_{7}$. The ionic radii of all elements are very similar so an empirical formula based on 13 atoms would be $\left(\mathrm{Fe}_{5.2} \mathrm{Ni}_{0.5} \mathrm{Mo}_{5.0} \mathrm{Ru}_{1.2} \mathrm{~W}_{0.3} \mathrm{Os}_{0.4} \mathrm{Ir}_{0.2} \mathrm{Pt}_{0.3}\right)$. The fcc RMN is euhedral. The unknown phase follows the grain boundary defined by the RMN and the melilite host. Which is consistent with a pseudomorph texture (Figure 5). This unknown phase was heavily deformed with elongate bladed crystal growths (Figure 5). TKD supported by TEM analyses indicated that the RMN was crystallographically aligned with the melilite host, as the [002] axis of the RMN was oriented parallel to the [201] axis of the melilite phase. The RMN was also crystallographically related to the unknown phase, sharing the same $<\mathrm{c}>$ axis to within $6^{\circ}$ (Figure 5). The RMN and the unknown phase 
had similar abundances of $\mathrm{Fe}, \mathrm{Ni}, \mathrm{Ru}$, Os and Ir. However, Mo was enriched within the unknown phase relative to the RMN, and $\mathrm{W}$ was present in the unknown phase but absent from the RMN. Comparing EDS measurements with TKD and TEM indicate that TEM is largely unaffected by Pt fluorescence unlike the TKD measurements. This implies that Pt was only present in the RMN and not in the unknown phase (Table 5).

Table 5: EDS measurements taken from Region ALH1, comparing TEM with TKD analyses. This label corresponds to the label in Figure 5p. b.d. = below detection limit: 0.5 at. \% Rh, 0.4 at. \% W, and 0.1 at. \% Pt.

\begin{tabular}{|c|c|c|c|c|c|c|c|c|c|c|}
\hline \multirow{2}{*}{ Technique } & \multirow{2}{*}{ Mineral } & \multicolumn{9}{|c|}{ at. $\%$} \\
\hline & & $\mathrm{Fe}$ & $\mathrm{Ni}$ & $\mathrm{Ru}$ & $\mathrm{Rh}$ & Mo & W & Os & $\mathrm{Ir}$ & $\mathrm{Pt}$ \\
\hline TEM & \multirow{2}{*}{$\mathrm{RMN}$} & 55.5 & 9.4 & 7.5 & b.d. & 6.7 & b.d. & 7.0 & 4.0 & 9.9 \\
\hline TKD & & 48.7 & 8.2 & 11.8 & 2.8 & 14.0 & b.d. & 2.0 & 2.8 & 9.8 \\
\hline TEM & \multirow{2}{*}{ unknown phase } & 50.9 & 9.4 & 6.3 & b.d. & 26.6 & 1.8 & 7.9 & 2.2 & b.d. \\
\hline TKD & & 40.0 & 3.7 & 9.2 & b.d. & 38.8 & 2.0 & 2.8 & 1.4 & 2.0 \\
\hline
\end{tabular}

\subsection{Vigarano - region V1}

TKD analyses of Vigarano - region V1 revealed a large anhedral $1 \mu \mathrm{m}$ RMN with a fcc structure associated with an isolated spinel grain to the left of the section. To the right of the section, there was a cluster of 7 RMNs between $0.5-0.1 \mu \mathrm{m}$ (Figure 6). The cluster of RMNs were euhedral fcc crystals with twinning planes which rotated approximately $60^{\circ}$ around the [111] axis with a $20-130 \mathrm{~nm}$ spacing between twin planes (Figure 6). The host phase of the RMNs was anorthite feldspar which was also twinned with a $180^{\circ}$ rotation around the twin plane (Figure 6). Each RMN had a different composition, with variations observed across all elements despite being located in the same inclusion of the same meteorite, and even between the cluster of 7 RMNs exhibiting twinning (Table6).

\subsection{Vigarano - region V2}

TKD analyses of Vigarano - region V2 revealed a RMN and associated metal and oxide phases hosted within a spinel grain. The boundary with the spinel and the assemblage exhibited straight edges. The assemblage was subdivided into three phases: a RMN hcp phase, powellite, and awaruite. Each phase had a different composition. Mo only found in the powellite. The awaruite exhibited twinning with a rotation about the [111] axis of $60^{\circ}$ (Figure 7). Os only occurred in the RMN phase which was also enriched in Ru and depleted in Ni relative to the awaruite (Table 7).

\section{Discussion}

Previous studies of RMNs have mostly lacked high resolution in situ crystallographic measurements, and instead focussed on the compositional variability between RMNs and mineralogical associations to inform 


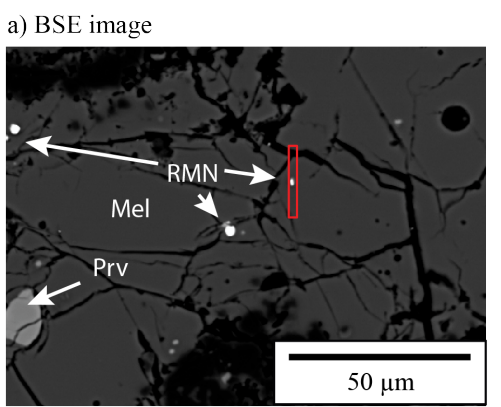

c) HRTEM image

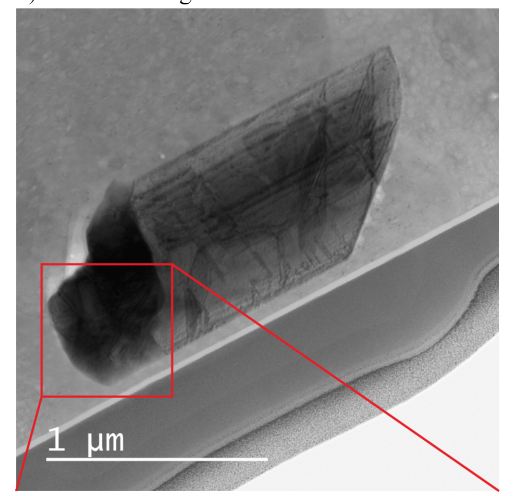

d) HRTEM image

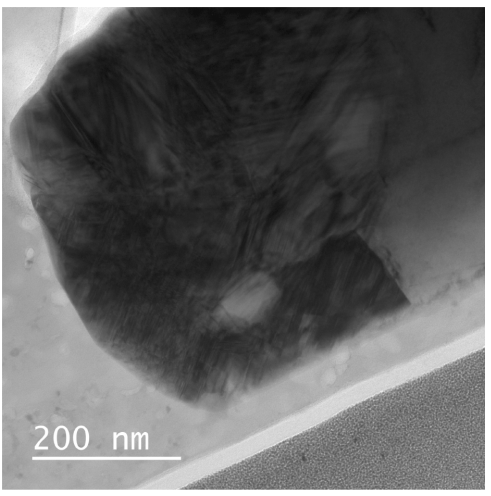

b) TKD band contrast image

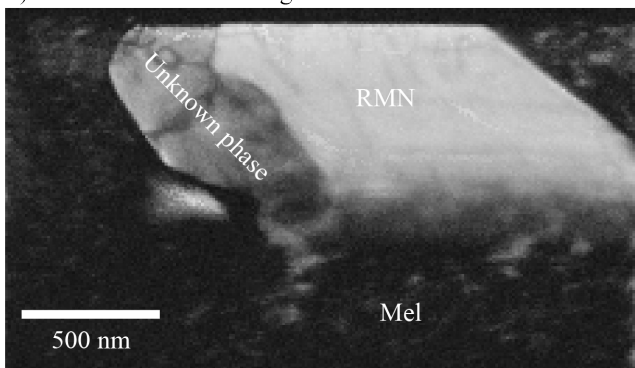

e) TKD phase map

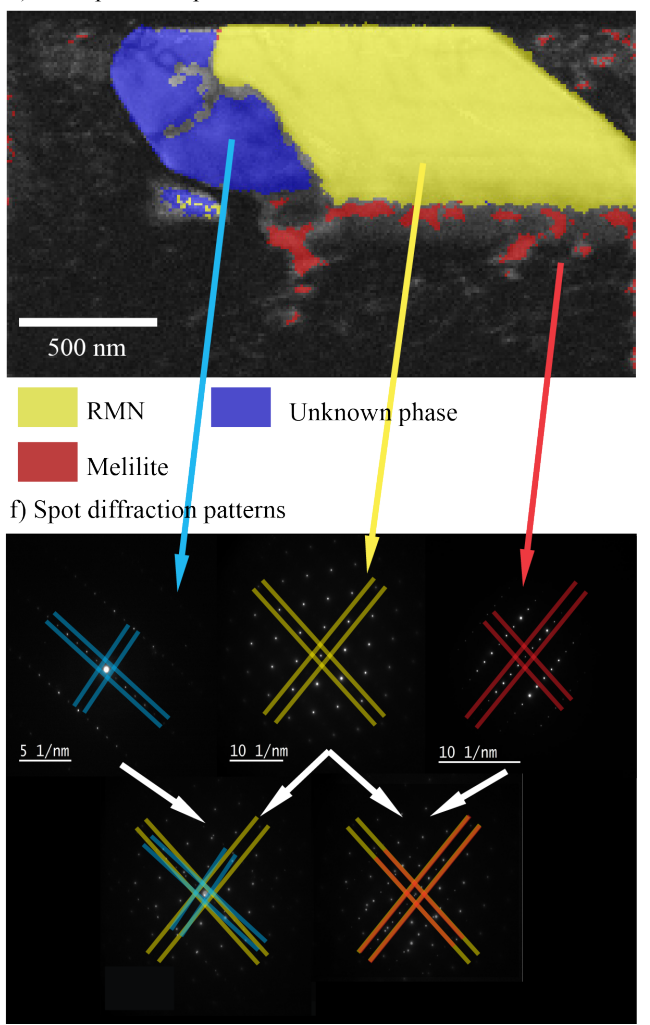

Figure 5: Characteristics of ALH 77307 - region ALH1: TKD analyses from a FIB-produced TEM lamellae of an RMN bearing melilite crystal within a Type B-like CAI. a) A BSE image of the RMN in situ, prior to FIB extraction, hosted within a Type B-like CAI. Associated minerals are melilite (Mel) and perovskite (Prv), b) TKD band contrast image of the RMN and associated unknown phase. The labels indicate where EDS spectra from TKD and TEM were taken in Table 5 c) TEM image of the RMN and associated unknown phase indicating the RMN has several dislocation planes, d) TEM zoomed image of the unknown phase indicating the phase is heavily dislocated, exhibiting oriented bladed crystal morphologies, e) TKD phase map indicating a euhedral RMN (yellow), its associated unknown phase (blue), and the melilite host (red). The RMN is observed to be euhedral and the unknown phase adopts a pseudomorph texture with the RMN, f) TEM spot diffraction patterns taken from each phase. These spot diffraction patterns are overlain which revealed a shared $<\mathrm{c}>$ crystal axis between the RMN and the unknown phase within $6^{\circ}$. The RMN [002] axis is shared with the [201] axis of the melilite host. 

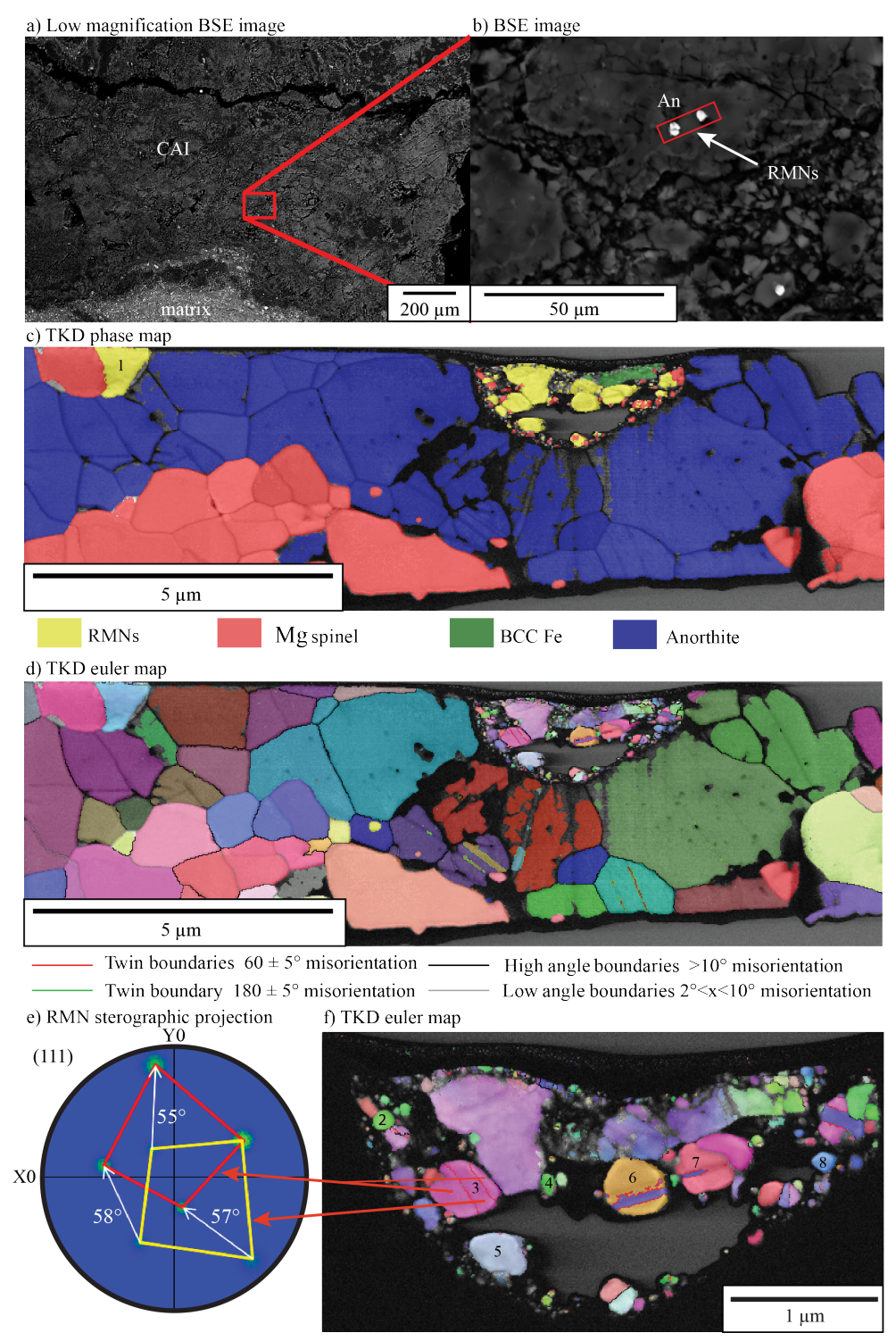

Figure 6: Characteristics of Vigarano - region V1: TKD analyses of a large RMN associated with spinel and a cluster of twinned RMNs in a void. These RMNs are hosted in anorthite contained within the altered mantle of a fluffy-type A CAI. a) A lower magnification BSE image showing the wider context of region V1 within the CAI, b) A BSE image of region V1 prior to sample extraction indicating two RMNs (bright spots). The red rectangle indicates the orientation of the extracted TEM section. The RMNs here are associated with anorthite (An), c) TKD phase map indicating RMNs in yellow. The large RMN to the left of the section is associated with spinel (red). The number indicated the EDS measurement in Table 6 The RMN cluster to the right is within a void encapsulated by anorthite (blue), d) TKD Euler image of the section indicating crystallographic orientations. Grain boundaries are noted by black lines and twin planes are noted in red. The anorthite exhibits twins rotating at $180^{\circ}$ around the twin plane, e) Stereographic projection plotting the poles to the crystal face for a twinned RMN indicating a rotation about the [111] axis of $60^{\circ}, \mathrm{f}$ ) A TKD euler image magnified at the right hand side RMN cluster revealing twin planes (red lines) within the RMNs. The numbers indicate the location of EDS measurements detailed in Table 5 
Table 6: EDS measurements taken from region V1. The RMN number corresponds to the numbered regions of Figures 6r and e. b.d. = below detection limit: 1 at. $\%$ Mo, 0.2 at. $\%$ Ir and 0.1 at. \% Pt.

\begin{tabular}{cccccccc}
\hline \multirow{2}{*}{ RMN } & \multicolumn{7}{c}{ at. $\%$} \\
& Fe & Ni & Ru & Mo & Os & Ir & Pt \\
\hline 1 & 48.7 & 48.6 & 1.8 & b.d. & 0.4 & b.d & 0.5 \\
2 & 67.4 & 15.5 & 11.8 & b.d. & 2.5 & 2.8 & b.d. \\
3 & 62.9 & 29.4 & 5.6 & b.d. & 1.1 & 0.9 & b.d. \\
4 & 65.6 & 14.2 & 14.2 & b.d. & 2.4 & 3.6 & b.d. \\
5 & 61.4 & 30.1 & 4.6 & b.d. & 1.0 & 1.9 & 0.9 \\
6 & 55.3 & 39.2 & 2.5 & b.d. & 0.7 & 1.0 & 1.4 \\
7 & 57.0 & 36.4 & 3.5 & b.d. & 1.1 & 1.1 & 0.9 \\
8 & 64.3 & 17.8 & 10.7 & b.d. & 3.3 & 3.8 & b.d. \\
\hline
\end{tabular}

Table 7: EDS measurements taken from region V2. The label corresponds to the label regions of Figure 7 . b.d. = below detection limit: 1 at. $\% \mathrm{Ni}, 0.4$ at. $\% \mathrm{Ru}, 1$ at. $\% \mathrm{Mo}, 0.1$ at. $\%$ Os, 0.2 at. $\%$ Ir, 0.1 at. $\%$ Pt, 0.1 at. $\%$ Ca and 1 at. $\%$ O. The powellite composition was derived using calculated oxygen contents of powellite, not the measured abundances from the EDS measurement

\begin{tabular}{cccccccccc} 
RMN & \multicolumn{1}{c}{ at. $\%$} & & & & \\
& Fe & Ni & Ru & Mo & Os & Ir & Pt & Ca & O \\
\hline Powellite 1 & 1.4 & b.d. & b.d. & 17.8 & b.d. & b.d. & 0.4 & 13.8 & 66.7 \\
Powellite 2 & 1.2 & b.d. & b.d. & 18.0 & b.d. & b.d. & b.d. & 14.2 & 66.7 \\
Awaruite & 42.3 & 41.8 & 8.5 & b.d. & b.d. & 3.9 & 3.6 & b.d. & b.d. \\
RMN & 45.2 & 8.4 & 35.1 & b.d. & 6.8 & 3.3 & 1.2 & b.d. & b.d. \\
\hline
\end{tabular}


a) Low magnification BSE imageb) BSE image

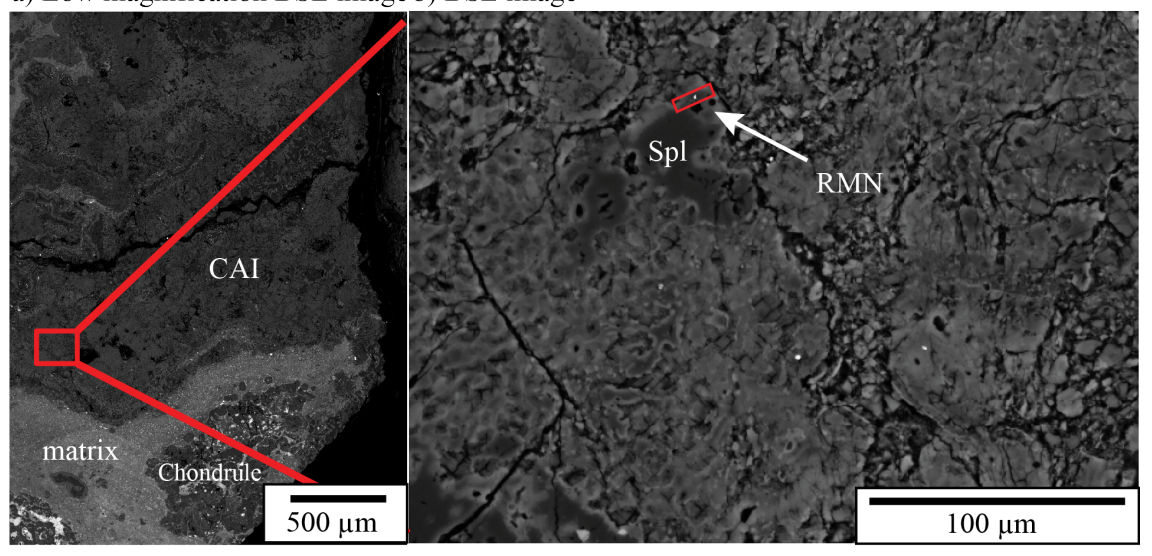

c) TKD phase map

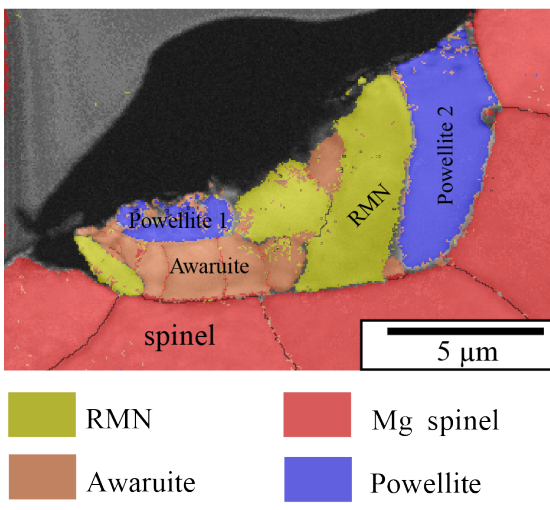

e) Awaruite stereographic projection

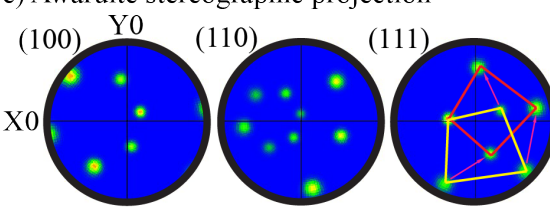

f) Powellite stereographic projection

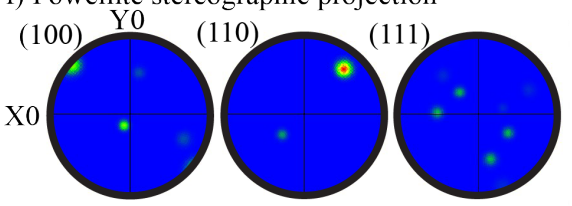

d) TKD euler image

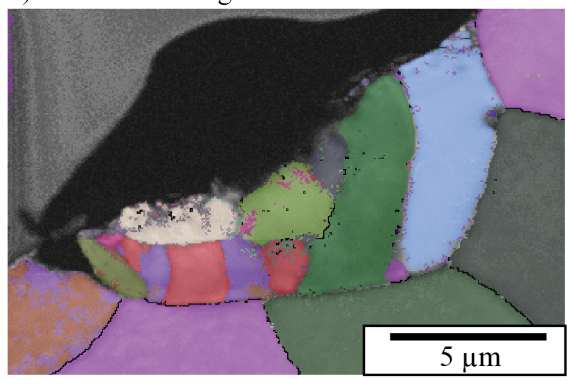

Twin boundaries $60 \pm 5^{\circ}$ misorientation

Grain boundaries $>10^{\circ}$ misorientation g) RMN steregraphic projection

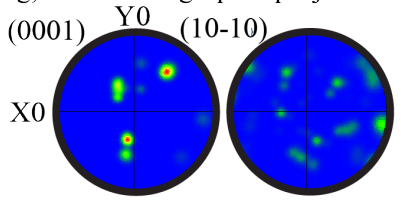

h) Spinel stereographic projection

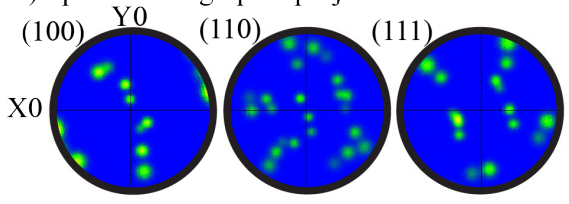

Figure 7: Characteristics of Vigarano - region V2. TKD analyses of RMNs and associated HSE alteration phases. These RMNs are hosted in spinel contained within the altered mantle of a fluffy type A CAI. a) Low magnification BSE image of region V2 in the wider context of the CAI; also showing a nearby matrix region and chondrule (Chond), b) A BSE image of the RMN (bright spot) in context, before extraction. The red rectangle indicates the orientation of the TEM section, the RMN here is associated with spinel (Spl), c) A TKD phase map of the section indicating the RMN (green) powellite (red) and awaruite (blue) assemblage hosted in a spinel (yellow). The labels indicate where EDS spectra were taken in Table 7 d) A TKD euler map indicating crystallographic orientations of all the phases. Twin planes are shown as red lines indicating the awaruite phase is twinned, e) Lower hemisphere stereographic projection of crystallographic orientations of the awaruite, indicating a rotation about the [111] axis of $\left.60^{\circ}, \mathrm{f}\right)$ Lower hemisphere stereographic projection of crystallographic orientations of the powellite, g) Lower hemisphere stereographic projection of crystallographic orientations of the two largest RMN grains with a shared pole in the [110] axis, h) Lower hemisphere stereographic projection of crystallographic orientations of the spinel. There are no systematic CORs observed between any of the phases. 
interpretations. This new high resolution data provided by TKD may provide us with the key to accurately interpret the formation history of individual RMNs and any subsequent alteration, based on a synthesis of their petrographic context, morphology, chemistry, and CORs with host phases.

Similar analyses have been applied successfully to other phases (e.g. Jacob et al. 2016 and references

\subsection{Allende - region A1}

n RMNs are mostly interpreted as a primary phase (Berg et al., 2009, Schwander et al., 2015b, Palme \& Wlotzka, 1976). However, if the RMNs observed here represent preserved primary phases they must survive chondrule formation, and subsequent sulphidation either in the nebula (Lauretta et al., 1996) or during metasomatism on the CV parent body (Blum et al., 1989) to arrive in their current petrographic context (Figure 1 a). The shared COR between RMNs suggests that these RMNs are genetically related. Furthermore, these RMNs are depleted in W, Mo and Os which are readily mobilised during oxidation and sulphidation of RMNs (Fegley \& Palme, 1985, Jones \& Scott, 1996, Palme et al., 1998). These observations suggest that these RMNs were affected if not formed by secondary processes either in the nebula or on the CV parent body. Therefore, a primordial origin for these RMNs is unlikely.

Deriving RMNs through secondary processes within chondrules requires a concentration mechanism for the HSEs due to their low abundances (measurements of bulk chondrules of the Allende meteorite indicate that they are depleted relative to bulk CV in siderophile elements such as Ir although coarse grained rims may be enriched or consistent with bulk CV (Rubin \& Wasson, 1987; Grossman et al., 1988, Lodders, 2003), a mechanism which produces and preserves the COR between RMNs and forsterite (Figure 2), and generates heterogeneous Ir and Pt compositions (Table 2).

HSE are soluble in sulphide (Makovicky et al., 1986, 1988, 1990). However, as RMNs are present the environmental conditions must have promoted the exsolution or preservation of metal. HSE alloys have been shown to exsolve from HSE-saturated sulphides during cooling as the solubility of HSE in sulphide decreases at low temperatures (Makovicky et al., 1986, 1988). HSE alloys can also be formed by S-loss from the system (Peregoedova et al. 2004) due to a reduction in the solubility of HSEs at low oxygen fugacity (Ballhaus \& Ulmer, 1995) even at trace levels of HSE (Peregoedova et al. 2004). If these RMNs were exsolution products during cooling of the sulphide we might expect to observe a COR with the host sulphide (Feinberg et al. 2004, Fleet et al. 1980 which is not the case here.

The sulphide nodules within the chondrule are all rounded blebs which is consistent with the formation of a metal-silicate immiscible fluid during chondrule formation and melting (Grossman \& Wasson, 1982). 
This would also have the effect of concentrating the HSEs in the metal phase due to their high partition coefficients ( $\mathrm{D}^{\text {metal} / \text { silicate }}$, of approximately $10^{4}$ (Grossman, 2010, Borisov \& Palme, 1995, 1997)). Some of the sulphide blebs exhibit rim structures which are consistent with the expected textures produced by nebula sulphidation (Lauretta et al., 1996) and implies that these blebs were once originally pure metal. However, this appears to be the only sulphide bleb to contain RMNs in the chondrule. This suggests that the HSEs were not initially evenly distributed throughout the chondrule, as in that case all or none of the sulphide blebs should contain RMNs. This suggests that the HSEs were pre-concentrated, likely in an RMN, which was assimilated into a single metal bleb. Thus, enriching this bleb in HSEs.

During sulphidation of the chondrule (Lauretta et al., 1996) the sulphur fugacity of the vapour was insufficient to promote the incorporation of $\mathrm{Pt}$ and $\mathrm{Ir}$ into the sulphide causing it to be retained in the metal. These RMNs are then the product of the migration of HSEs which are more compatible with the metal phase (Ir, Pt) away from the sulphidation front. Experimental work by Peregoedova et al. (2004) suggest that this may occur at sulphur fugacity between $f S_{2}-6.9$ and -0.9 . If these could be constrained further, we could determine the maximum $f S_{2}$ experienced during nebula sulphidation. The heterogeneous chemistry is generated by either heterogeneous distribution of HSEs in the nodule, which is unlikely due to the affinity of HSE to metal (Borisov \& Palme, 1995, 1997; Grossman, 2010), or heterogeneities in local mineral chemistry - proximity to forsterite or proximity to troilite etc, or variable sizes of the 'catchment area' from which the HSEs were delivered to the RMN.

The COR between the forsterite grains and RMNs by way of a shared [100] axis may originate during the initial crystallisation of the chondrule, where forsterite crystallised around the Fe-metal bleb. Subsequent crystallisation of the Fe-metal would produce a COR by way of minimising the interfacial energy (Frondel, 1940), see also reaction fabric (Lauder, 1961)). Therefore, these RMNs preserve the initial alignment of the original Fe-metal bleb. There is no possibility of producing the observed alignment by compaction or through magnetic susceptibility. Furthermore, these RMNs are not connected in a 3D worm-like structure but are isolated grains, so the original alignment is preserved despite the isolation of these RMNs. This orientation relationship is preserved during sulphidation (Lauretta et al., 1996), and throughout any subsequent metasomatic alteration of the CV3 parent body (Blum et al., 1989).

In summary, the most likely sequence of events that occurred to produce the RMNs in Allende - region A1 is as follows: during chondrule formation an immiscible metal-silicate melt formed (Grossman \& Wasson, 1982). One of these metal nodules incorporated an HSE enriched particle. On cooling the forsterite crystallised first around the still molten metal nodule. Further cooling crystallised the metal nodule with a COR with forsterite through minimising the interfacial energy (Frondel, 1940, Lauder, 1961). The chondrule was then exposed to a sulphur-rich gas which sulphidised the metal grain (Lauretta et al., 1996). The sulphur fugacity was such that Pt and Ir were above the saturation point for the sulphide (Peregoedova et al., 2004) causing them to preferentially remain in the metal. Tungsten, Mo and Os which have higher affinities for sulphide remained in the sulphide (Fegley \& Palme, 1985) or perhaps were stripped during metasomatic 
alteration on the parent body (Blum et al. 1989).

\subsection{Allende - region A2} of the two phases under oxidising conditions (Fegley \& Palme, 1985). Therefore, this assemblage and the 
associated RMNs may represent exsolution products during the phase separation of the initially homogeneous alloy at lower temperatures under variable oxygen fugacity (Palme et al., 1994, Blum et al., 1988, Blum

335 indicate that is was subsequently annealed (Smith, 1948; Komatsu et al., 2001), consistent with this type of 
inclusion (MacPherson et al., 2005, Grossman et al., 2000) The RMN is associated with an unknown phase which adopted a pseudomorph texture with the RMN. For metal grains in meteorites a euhedral morphology although it is plausible that euhedral metal laths could grow during metamorphism none have been reported (Rubin, 1994). Therefore, euhedral RMNs are interpreted to have grown in an unconstrained environment.

The unknown phase was likely to be a secondary phase formed by alteration of the RMN, separating Mo and $\mathrm{W}$ into the unknown phase due to their increased mobility during alteration events Fegley \&

\subsection{Vigarano - region V1 and V2}

The CAI bearing the V1 and V2 regions has a zoned texture with a relatively unaltered core. There is potentially re-heated material surrounding the core, and Wark Lovering rims around the edge of the CAI. 
The region is interpreted as 're-heated' due to the presence of annealing textures such as $180^{\circ}$ mirror twins within the anorthite (Figure 6) and $120^{\circ}$ grain-boundary-intersection spinel grains (Figure 7) (Smith, 1948 ,

405 awaruite in V2 (Figure 7) have not previously been reported in RMNs despite numerous and detailed SEM (Sylvester et al., 1990; Schwander et al. 2015b, Wark \& Lovering, 1976) and TEM studies (Harries et al. 2012, Croat et al. 2013). This demonstrates the value of TKD and its ability to map the crystallography and chemistry of a lamella rapidly, in comparison to established techniques such as TEM. Twinning can be 435 within an anorthite grain (Region V1) and a spinel grain (Region V2).

The RMNs in V1 had two distinct morphologies: an anhedral RMN associated with spinel, and several euhedral twinned RMNs with straight grain boundaries (Figure 66). The euhedral RMNs likely formed in an unconstrained environment in the presence of a fluid or gas (Rubin 1994). Their accumulation in a void space indicates that they may have originally been free floating in the nebula gas, or were initially suspended in a mineral which has since been removed. The chemistry of each RMN in V1 are different from each other, and none have chondritic relative elemental ratios even though these grains are hosted within the same CAI, and in some places are situated adjacent to each other. However, an 'average' RMN derived from the mean HSE abundance of these RMNs is consistent with chondritic elemental ratios. None contain detectable Mo suggesting these elements may have been remobilised (Fegley \& Palme, 1985). There is no transfer or re-equilibration of HSEs, even between RMNs separated by only a few nanometres. The RMN associated with the spinel in V1 together form a spherical inclusion. There is a slight curvature of the RMN grain boundary towards the bottom of the inclusion (Figure 6). This curvature could be interpreted as the meniscus between two fluids which suggests that the RMN and the spinel were both molten forming an immiscible silicate-metal melt.

The region V2 contains a complex HSE-rich inclusion and is unlike any previously described RMN in this study. The RMN has separated into three phases: a Mo-rich powellite, a Ni-rich twinned awaruite and an Os, Ru-enriched RMN. These textures are consistent with an OA (El Goresy et al., 1977; Blum et al. 1988) (Figure 7). This inclusion contains two metal phases: awaruite and the RMN, which exhibit a complementary depletion or enrichment in $\mathrm{Ru}$ and an enrichment or depletion in $\mathrm{Ni}$, respectively. The powellite is the only phase present to contain Mo. The redox state of Mo implies the event that mobilised the Mo was oxidising (Fegley \& Palme, 1985) and likely occurred within the CAI which provided the Ca component. This containment in the CAI inhibited the escape of Mo. All of the phases exhibit deviations from chondritic relative abundances consistent with a OA (El Goresy et al., 1977, Palme et al., 1994).

The crystal twins observed within RMNs in Vigarano in both the euhedral RMNs in V1 (Figure 6) and the produced via a number of different mechanisms, such as growth, annealing and deformation (Cahn, 1954).

The pressure and strain rate required for the onset of shock twinning for micrometre alloys of HSEs such as RMNS is not well constrained. The Vigarano meteorite has undergone very little processing in terms of deformation and shock (Scott et al. 1992). Vigarano is classified as shock stage S1 (Komatsu et al. 
2001; Scott et al., 1992) implying it experienced <5 GPa (Stöffler \& Keil, 1991). Neumann lines in kamacite

440

kamacite, but such textures have not been reported within these metal inclusions in Vigarano (McSween, 1977). Furthermore, shock twinning is dependent on both grain size, where smaller grains are more resistant to shock twinning (Meyers et al. 1995). Therefore, if RMNs are comparable to kamacite metals in Vigarano and the fact that a second RMN within the same TEM lamellae does not exhibit twinning it is reasonable to assume that these twins are not derived from shock related processes.

This leaves two possibilities: growth twins and annealing twins. If the observed twins were growth twins formed from nucleation these would be observed as simple penetration twins. This would result in clear crystal faces with changes in the morphology of the RMN which are coincident with the twin boundary. This is not observed within the sample as the crystal faces are well formed and continuous across the twin boundary. We would also expect this to be a much more common observation in RMNs if they formed growth twins.

Annealing twins form at high temperatures with relatively little stress. In fcc crystals they classically form along the [111] axis with a $60^{\circ}$ misorientation (Brandon, 1966), which is consistent with the observed RMN twins in Vigarano (Figure 6). This implies that the twinning observed is probably formed by annealing. TEM experiments by Dai et al. (2001) observed annealing twin formation in FePt nanoparticles. This study indicated that while heating under vacuum for one hour, annealing twins began to form at $450{ }^{\circ} \mathrm{C}$, and were completely annealed by $530{ }^{\circ} \mathrm{C}$. The nanoparticles studied coalesced into larger grains at $600-700{ }^{\circ} \mathrm{C}$. The RMNs observed in Vigarano, however, have not coalesced. The specific grains in the aforementioned study are compositionally different to RMNs observed in our research, so only limited caparisons can be made. However, the temperature dependence of the onset of annealing twinning (Dai et al., 2001) indicates that by constraining their formation temperature by experiment for RMN alloy compositions, annealing twins could be used as a coarse thermometer for early Solar System processes.

These annealing twins could form either as a result of thermal metamorphism on the CV parent body, or pre-accretion in the nebula either as free floating RMNs or subsequent to RMN incorporation into the CAI. 口

The CAI here did contain nepheline and sodalite which are evidence for parent body alteration Krot et al. 1997). The highest estimates place the maximum parent body temperatures experienced by Vigarano at $415{ }^{\circ} \mathrm{C}$ (Cody et al. 2008), and most estimates for Vigarano peak temperatures are much lower than this $\left(<330{ }^{\circ} \mathrm{C}\right)$ (e.g. Bonal et al. 2006, 2007). If RMNs anneal in this temperature range we could further constrain parent body temperatures. The twinning present in the awaruite in V2 may be of this nature as awaruite is a low temperature phase which forms at $<500{ }^{\circ} \mathrm{C}$ (Reuter et al., 1989) associated with parent body alteration (Müller et al. 1995).

These RMNs are hosted in anorthite and spinel which do not exhibit textures consistent with melting. These phases melt at $1250{ }^{\circ} \mathrm{C}$ and $1550{ }^{\circ} \mathrm{C}$ respectively (Stolper, 1982) so therefore, if RMNs form annealing twins between $400-1200{ }^{\circ} \mathrm{C}$ (the maximum Vigarano parent body temperature and the temperature that 
The relative chemical abundances of HSEs in RMNs are variable with the exception of the small spherical RMNs in A2 which have uniform chemistry with near chondritic relative elemental abundances. This may be due to a separation of immiscible metal-silicate fluids during melting of an OA. These RMNs are also 

complex formation history. These observations are consistent with the suggestion of Daly et al. (this issue), that some RMNs have survived Solar System formation and preserve an inherited heterogeneity from the Giant Molecular Cloud. 
Berg, T., Maul, J., Schönhense, G., Marosits, E., Hoppe, P., Ott, U., \& Palme, H. (2009). Direct evidence for condensation in the early solar system and implications for nebular cooling rates. The Astrophysical Journal Letters, 702, L172-L176.

Bird, J. M., \& Bassett, W. A. (1980). Evidence of a deep mantle history in terrestrial osmium-iridium-

Bischoff, A., \& Palme, H. (1987). Composition and mineralogy of refractory-metal-rich assemblages from a Ca, Al-rich inclusion in the Allende meteorite. Geochimica et Cosmochimica Acta, 51, 2733-2748. 
Bischoff, A., \& Stoeffler, D. (1992). Shock metamorphism as a fundamental process in the evolution of planetary bodies: Information from meteorites. European Journal of Mineralogy, 4, 707-755.

Dai, Z., Sun, S., \& Wang, Z. (2001). Phase transformation, coalescence, and twinning of monodisperse FePt nanocrystals. Nano Letters, 1, 443-447.

Blum, J. D., Wasserburg, G., Hutcheon, I., Beckett, J., \& Stolper, E. (1989). Origin of opaque assemblages in C3V meteorites: Implications for nebular and planetary processes. Geochimica et Cosmochimica Acta, $53,543-556$.

Blum, J. D., Wasserburg, G. J., Hutcheon, I. D., Beckett, J. R., \& Stolper, E. M. (1988). 'Domestic' origin of opaque assemblages in refractory inclusions in meteorites. Nature, 331, 405-409.

Bonal, L., Bourot-Denise, M., Quirico, E., Montagnac, G., \& Lewin, E. (2007). Organic matter and metamorphic history of CO chondrites. Geochimica et Cosmochimica Acta, 71, 1605-1623.

Bonal, L., Quirico, E., Bourot-Denise, M., \& Montagnac, G. (2006). Determination of the petrologic type of CV3 chondrites by Raman spectroscopy of included organic matter. Geochimica et Cosmochimica Acta, $70,1849-1863$.

Borisov, A., \& Palme, H. (1995). The solubility of iridium in silicate melts: new data from experiments with Ir 10 Pt 90 alloys. Geochimica et Cosmochimica Acta, 59, 481-485.

Borisov, A., \& Palme, H. (1997). Experimental determination of the solubility of platinum in silicate melts. Geochimica et Cosmochimica Acta, 61, 4349-4357.

Brandon, D. (1966). The structure of high-angle grain boundaries. Acta metallurgica, 14, 1479-1484.

Cahn, R. (1954). Twinned crystals. Advances in Physics, 3, 363-445.

Campbell, A. J., Humayun, M., Meibom, A., Krot, A. N., \& Keil, K. (2001). Origin of zoned metal grains in the QUE94411 chondrite. Geochimica et Cosmochimica Acta, 65, 163-180.

Cody, G., Alexander, C. O., Yabuta, H., Kilcoyne, A., Araki, T., Ade, H., Dera, P., Fogel, M., Militzer, B., \& Mysen, B. (2008). Organic thermometry for chondritic parent bodies. Earth and Planetary Science Letters, 272, 446-455.

Croat, T., Berg, T., Bernatowicz, T., Groopman, E., \& Jadhav, M. (2013). Refractory metal nuggets within presolar graphite: First condensates from a circumstellar environment. Meteoritics 83 Planetary Science, $48,686-699$. 
Daly, L., Bland, P., Dyl, K., Forman, L., Evans, K., Trimby, P., Moody, S., Limei, Y., Liu, H., Ringer, S., Ryan, C., \& Saunders, M. (this issue). In situ analysis of Refractory Metal Nuggets in carbonaceous chodrites. Geochimica et Cosmochimica Acta, .

Eisenhour, D., \& Buseck, P. (1992). Transmission electron microscopy of RMNs: Implications for single-phase condensation of the refractory siderophile elements. Meteoritics, 27, 217-218.

El Goresy, A., Nagel, K., Dominik, B., \& Ramdohr, P. (1977). Fremdlinge: Potential presolar material in Ca-Al-rich inclusions of Allende. Meteoritics, 12, 215-216.

El Goresy, A., Nagel, K., \& Ramdohr, P. (1978). Fremdlinge and their noble relatives. Lunar and Planetary Science Conference Proceedings, 9, 1279-1303.

Fegley, B., \& Palme, H. (1985). Evidence for oxidizing conditions in the solar nebula from Mo and W depletions in refractory inclusions in carbonaceous chondrites. Earth and Planetary Science Letters, 72, $311-326$.

Feinberg, J. M., Wenk, H.-R., Renne, P. R., \& Scott, G. R. (2004). Epitaxial relationships of clinopyroxenehosted magnetite determined using electron backscatter diffraction (EBSD) technique. American Mineralogist, 89, 462-466.

Fleet, M. E., Bilcox, G. A., \& Barnett, R. L. (1980). Oriented magnetite inclusions in pyroxenes from the Grenville Province. Canadian Mineralogist, 18, 89.

Forman, L., Bland, P., Timms, N., Collins, G., Davison, T., Ciesla, F., Benedix, G., Daly, L., Trimby, P., Yang, L. et al. (2016). Hidden secrets of deformation: Impact-induced compaction within a CV chondrite. Earth and Planetary Science Letters, 452, 133-145.

Frondel, C. (1940). Oriented inclusions of staurolite, zircon and garnet in muscovite. Skating crystals and their significance. American Mineralogist, 25, 69-87.

Griffith, W. P. (2009). Melting the Platinum Group Metals. Platinum Metals Review, 53, 209-215.

Griffiths, T. A., Habler, G., \& Abart, R. (2016). Crystallographic orientation relationships in host-inclusion systems: New insights from large EBSD data sets. American Mineralogist, 101, 690-705.

Grossman, J. N., Rubin, A. E., Nagahara, H., \& King, E. A. (1988). Properties of chondrules. Meteorites and the early solar system, (pp. 619-659).

Grossman, J. N., \& Wasson, J. T. (1982). Evidence for primitive nebular components in chondrules from the Chainpur chondrite. Geochimica et Cosmochimica Acta, 46, 1081-1099.

Grossman, L. (1973). Refractory trace elements in Ca-Al-rich inclusions in the Allende meteorite. Geochimica et Cosmochimica Acta, 37, 1119-1140. 
Grossman, L. (2010). Vapor-condensed phase processes in the early solar system. Meteoritics $\&$ Planetary

Grossman, L., Ebel, D. S., Simon, S. B., Davis, A. M., Richter, F. M., \& Parsad, N. M. (2000). Major element chemical and isotopic compositions of refractory inclusions in C3 chondrites: The separate roles of condensation and evaporation. Geochimica et Cosmochimica Acta, 64, 2879-2894.

Harries, D., Berg, T., Langenhorst, F., \& Palme, H. (2012). Structural clues to the origin of refractory metal alloys as condensates of the solar nebula. Meteoritics \& Planetary Science, 47, 2148-2159.

Herlach, D. M. (1994). Non-equilibrium solidification of undercooled metallic metls. Materials Science and Engineering: R: Reports, 12, 177-272.

Hewins, R. H., Bourot-Denise, M., Zanda, B., Leroux, H., Barrat, J.-A., Humayun, M., Göpel, C., Greenwood, R. C., Franchi, I. A., Pont, S. et al. (2014). The Paris meteorite, the least altered CM chondrite so far. Geochimica et Cosmochimica Acta, 124, 190-222.

Hutcheon, I. D., Armstrong, J. T., \& Wasserburg, G. (1987). Isotopic studies of Mg, Fe, Mo, Ru and W in Fremdlinge from Allende refractory inclusions. Geochimica et Cosmochimica Acta, 51, 3175-3192.

Ivanenko, L., Behr, G., Spinella, C., \& Borisenko, V. (2002). RuSi 2: evidence of a new binary phase in the ruthenium-silicon system. Journal of crystal growth, 236, 572-576.

Jacob, D. E., Piazolo, S., Schreiber, A., \& Trimby, P. (2016). Redox-freezing and nucleation of diamond via magnetite formation in the Earth's mantle. Nature Communications, 7.

Jones, R., \& Scott, E. (1996). Chondrules and the protoplanetary disk. Cambridge University Press.

Keller, R. R., \& Geiss, R. H. (2012). Transmission EBSD from $10 \mathrm{~nm}$ domains in a scanning electron microscope. Journal of Microscopy, 245, 245-251.

Komatsu, M., Krot, A. N., Petaev, M. I., Ulyanov, A. A., Keil, K., \& Miyamoto, M. (2001). Mineralogy and petrography of amoeboid olivine aggregates from the reduced CV3 chondrites Efremovka, Leoville and Vigarano: Products of nebular condensation, accretion and annealing. Meteoritics $\mathscr{G}$ Planetary Science, $36,629-641$.

Krot, A. N., Scott, E. R., \& Zolensky, M. E. (1997). Origin of fayalitic olivine rims and lath-shaped matrix olivine in the CV3 chondrite Allende and its dark inclusions. Meteoritics \& Planetary Science, 32, 31-49.

Lauder, W. (1961). Reaction of crystal structures and reaction fabric. American Mineralogist, 46, 1317-1328.

Lauretta, D. S., Kremser, D. T., \& Fegley Jr, B. (1996). The rate of iron sulfide formation in the solar nebula. Icarus, 122, 288-315. 
Lodders, K. (2003). Solar system abundances and condensation temperatures of the elements. The Astrophysical Journal, 591, 1220-1247.

Ma, C., Beckett, J. R., \& Rossman, G. R. (2014). Allendeite $\left(\mathrm{Sc}_{4} \mathrm{Zr}_{3} \mathrm{O}_{12}\right)$ and hexamolybdenum (Mo, Ru, Fe), two new minerals from an ultrarefractory inclusion from the Allende meteorite. American Mineralogist, $99,654-666$.

MacPherson, G., Simon, S., Davis, A., Grossman, L., \& Krot, A. (2005). Calcium-Aluminum-Rich inclusions: Major unanswered questions. Chondrites and the protoplanetary disk, 341, 225.

Makovicky, E., Karup-Møller, S., Makovicky, M., \& Rose-Hansen, J. (1990). Experimental studies on the phase systems Fe-Ni-Pd-S and Fe-Pt-Pd-As-S applied to PGE deposits. Mineralogy and Petrology, 42, $307-319$.

Makovicky, M., Makovicky, E., \& Rose-Hansen, J. (1986). Experimental studies on the solubility and distribution of platinum group elements in base-metal sulphides in platinum deposits. Metallogeny of Basic and Ultrabasic Rocks, (pp. 415-425).

Makovicky, M., Makovicky, E., \& Rose-Hansen, J. (1988). Experimental evidence on the formation and mineralogy of platinum and palladium ore deposits. Mineral deposits within the European Community, (pp. 303-317).

Massalski, T. B., Okamoto, H., Subramanian, P., \& Kacprzak, L. (1990). Binary alloy phase diagrams. ASM International, 1990,, (p. 1485).

McSween, H. Y. (1977). Petrographic variations among carbonaceous chondrites of the Vigarano type. Geochimica et Cosmochimica Acta, 41, 1777-1790.

Mendybaev, R. A., Richter, F. M., \& Davis, A. M. (2006). Crystallization of melilite from CMAS-liquids and the formation of the melilite mantle of Type B1 CAIs: Experimental simulations. Geochimica et cosmochimica acta, 70, 2622-2642.

Meyers, M. A., Andrade, U. R., \& Chokshi, A. H. (1995). The effect of grain size on the high-strain, high-strain-rate behavior of copper. Metallurgical and materials transactions A, 26, 2881-2893.

Milholland, C. S., \& Presnall, D. C. (1998). Liquidus phase relations in the $\mathrm{CaO}-\mathrm{MgO}-\mathrm{Al}_{2} \mathrm{O}_{3}-\mathrm{SiO}_{2}$ system at 3.0 GPa: the aluminous pyroxene thermal divide and high-pressure fractionation of picritic and komatiitic magmas. Journal of Petrology, 39, 3-27.

Müller, W. F., Weinbruch, S., Walter, R., \& Müller-Beneke, G. (1995). Transmission electron microscopy of chondrule minerals in the Allende meteorite: constraints on the thermal and deformational history of granular olivine-pyroxene chondrules. Planetary and Space Science, 43, 469-483. 
Nagahara, H., Kushiro, I., \& Mysen, B. O. (1994). Evaporation of olivine: Low pressure phase relations of the olivine system and its implication for the origin of chondritic components in the solar nebula. Geochimica et cosmochimica acta, 58, 1951-1963.

Palme, H., Borisov, A., \& Wulf, A. (1998). Experimental determination of the oxidation sequence of refractory metals. Lunar and Planetary Science Conference, 29, 1611.

Palme, H., Hutcheon, I., \& Spettel, B. (1994). Composition and origin of refractory-metal-rich assemblages in a Ca, Al-rich Allende inclusion. Geochimica et cosmochimica acta, 58, 495-513.

Palme, H., Kurat, G., Spettel, B., \& Burghele, A. (1989). Chemical composition of an unusual xenolith of the Allende meteorite. Zeitschrift für Naturforschung A, 44, 1005-1014.

Palme, H., \& Wlotzka, F. (1976). A metal particle from a Ca, Al-rich inclusion from the meteorite Allende, and the condensation of refractory siderophile elements. Earth and Planetary Science Letters, 33, 45-60.

Peregoedova, A., Barnes, S.-J., \& Baker, D. R. (2004). The formation of Pt-Ir alloys and Cu-Pd-rich sulfide melts by partial desulfurization of $\mathrm{Fe}-\mathrm{Ni}-\mathrm{Cu}$ sulfides: results of experiments and implications for natural systems. Chemical Geology, 208, 247-264.

Piazolo, S., La Fontaine, A., Trimby, P., Harley, S., Yang, L., Armstrong, R., \& Cairney, J. M. (2016). Deformation-induced trace element redistribution in zircon revealed using atom probe tomography. Nature communications, $\%$.

Presnall, D., Dixon, S. A., Dixon, J. R., O’Donnell, T., Brenner, N., Schrock, R., \& Dycus, D. (1978). Liquidus phase relations on the join diopside-forsterite-anorthite from $1 \mathrm{~atm}$ to $20 \mathrm{kbar}$ : their bearing on the generation and crystallization of basaltic magma. Contributions to Mineralogy and Petrology, 66, 203-220.

Reuter, K., Williams, D. B., \& Goldstein, J. (1989). Determination of the Fe- Ni phase diagram below $400^{\circ} \mathrm{C}$. Metallurgical Transactions A, 20, 719-725.

Rubin, A. E. (1994). Euhedral tetrataenite in the Jelica meteorite. Mineralogical Magazine, 58, 215-222.

Rubin, A. E., \& Wasson, J. T. (1987). Chondrules, matrix and coarse-grained chondrule rims in the Allende meteorite: Origin, interrelationships and possible precursor components. Geochimica et Cosmochimica Acta, 51, 1923-1937.

Rudraswami, N., Prasad, M. S., Plane, J., Berg, T., Feng, W., \& Balgar, S. (2014). Refractory metal nuggets in different types of cosmic spherules. Geochimica et Cosmochimica Acta, 131, 247-266.

Schwander, D., Berg, T., Harries, D., Schönhense, G., \& Ott, U. (2014). Composition and clues to the origin of refractory metal nuggets extracted from chondritic meteorites. Meteoritics 83 Planetary Science, 49, $1888-1901$. 
Schwander, D., Buhre, S., Schönhense, G., \& Ott, U. (2015a). Synthesis of refractory metal nuggets and constraints on the thermal histories of nugget-bearing $\mathrm{Ca}$, Al-rich inclusions. Meteoritics $6 \mathcal{G}$ Planetary Science, 50, 893-903.

735

Schwander, D., Kööp, L., Berg, T., Schönhense, G., Heck, P., Davis, A., \& Ott, U. (2015b). Formation of refractory metal nuggets and their link to the history of CAIs. Geochimica et Cosmochimica Acta, 168, $70-87$.

Scott, E. R., Keil, K., \& Stöffler, D. (1992). Shock metamorphism of carbonaceous chondrites. Geochimica et Cosmochimica Acta, 56, 4281-4293.

Smith, C. (1948). Grains, Phases, and Interfaces: an Interpretation of Microstructure. Transactions of the American Institute of Mining, Metallurgical, and Petroleum Engineers, 175, 15-51.

Sneddon, G. C., Trimby, P. W., \& Cairney, J. M. (2016). Transmission Kikuchi diffraction in a scanning electron microscope: a review. Materials Science and Engineering: R: Reports, 110, 1-12.

Stöffler, D., \& Keil, K. (1991). Shock metamorphism of ordinary chondrites. Geochimica et Cosmochimica Acta, 55, 3845-3867.

Stolper, E. (1982). Crystallization sequences of Ca-Al-rich inclusions from Allende: An experimental study. Geochimica et Cosmochimica Acta, 46, 2159-2180.

Sylvester, P. J., Ward, B. J., Grossman, L., \& Hutcheon, I. D. (1990). Chemical compositions of siderophile element-rich opaque assemblages in an Allende inclusion. Geochimica et Cosmochimica Acta, 54, 34913508.

Trimby, P. W. (2012). Orientation mapping of nanostructured materials using transmission Kikuchi diffraction in the scanning electron microscope. Ultramicroscopy, 120, 16-24.

Trimby, P. W., Cao, Y., Chen, Z., Han, S., Hemker, K. J., Lian, J., Liao, X., Rottmann, P., Samudrala, S., Sun, J. et al. (2014). Characterizing deformed ultrafine-grained and nanocrystalline materials using transmission Kikuchi diffraction in a scanning electron microscope. Acta materialia, 62, 69-80.

Wang, Y., Hua, X., \& WeiBiao, H. (2007). Petrogenesis of opaque assemblages in the Ningqiang carbonaceous chondrite. Science in China Series D: Earth Sciences, 50, 886-896.

Wark, D. (1986). Evidence for successive episodes of condensation at high temperature in a part of the solar nebula. Earth and planetary science letters, 77, 129-148.

Wark, D., \& Lovering, J. (1976). Refractory/platinum metal grains in Allende calcium-aluminium-rich clasts (CARC's): possible exotic presolar material? Lunar and Planetary Science Conference, 7, 912. 